\title{
Sex, drugs, and bitcoin: How much illegal activity is financed through cryptocurrencies? ${ }^{*}$
}

\author{
Sean Foley \\ University of Sydney \\ Jonathan R. Karlsen \\ University of Technology Sydney \\ Tālis J. Putniñ̌s \\ University of Technology Sydney \\ Stockholm School of Economics in Riga
}

Cryptocurrencies are among the largest unregulated markets in the world. We find that approximately one-quarter of bitcoin users are involved in illegal activity. We estimate that around $\$ 76$ billion of illegal activity per year involves bitcoin (46\% of bitcoin transactions), which is close to the scale of the US and European markets for illegal drugs. The illegal share of bitcoin activity declines with mainstream interest in bitcoin and with the emergence of more opaque cryptocurrencies. The techniques developed in this paper have applications in cryptocurrency surveillance. Our findings suggest that cryptocurrencies are transforming the black markets by enabling "black e-commerce". (JEL G18, O31, O32, O33)

\footnotetext{
${ }^{*}$ We thank two anonymous referees, the RFS FinTech sponsoring editors (Itay Goldstein, Wei Jiang, and Andrew Karolyi), Michael Weber (discussant), David Easley, Maureen O'Hara, Paolo Tasca, as well as the conference/seminar participants at the RFS FinTech Conference, the RFS FinTech Workshop of Registered Reports, the Behavioral Finance and Capital Markets Conference, the UBS Equity Markets Conference, the University of Technology Sydney, the Australian Federal Police, AUSTRAC, and the Financial Conduct Authority (UK) for useful comments and suggestions. We also thank Tristan Blakers, Adrian Manning, Luke Anderson, Yaseen Kadir, Evans Gomes, and Joseph Van Buskirk for assistance relating to data. Jonathan Karlsen acknowledges financial support from the Capital Markets Co-operative Research Centre. Tālis Putniņš acknowledges financial support from the Australian Research Council (ARC) under grant number DE150101889. The Online Appendix that accompanies this paper can be found at goo.gl/GvsERL.

Send correspondence to Tālis Putniņš, UTS Business School, University of Technology Sydney, PO Box 123 Broadway, NSW 2007, Australia; telephone: +61 2 95143088. Email: talis.putnins@uts.edu.au.
} 
Cryptocurrencies have grown rapidly in price, popularity, and mainstream adoption. Over 1,800 cryptocurrencies exist with market capitalization exceeding $\$ 300$ billion as at July 2018. Bitcoin, the largest cryptocurrency, accounts for around half of the total market capitalization. The numerous online cryptocurrency exchanges and markets have daily dollar volume of around $\$ 50$ billion. ${ }^{1}$ Over 170 "cryptofunds" have emerged (hedge funds that invest solely in cryptocurrencies), attracting around \$2.3 billion in assets under management. ${ }^{2}$ Recently, bitcoin futures have commenced trading on the CME and CBOE, catering to institutional demand for trading and hedging bitcoin. ${ }^{3}$ What was once a fringe asset is quickly maturing.

The rapid growth in cryptocurrencies and the anonymity that they provide users has created considerable regulatory challenges. An application for a \$100 million cryptocurrency Exchange Traded Fund (ETF) was rejected by the US Securities and Exchange Commission (SEC) in March 2017 (and several more rejected in 2018) amid concerns including the lack of regulation. The Chinese government banned residents from trading cryptocurrencies and made initial coin offerings (ICOs) illegal in September 2017. Central bank heads, such as the Bank of England's Mark Carney, have publicly expressed concerns about cryptocurrencies. While cryptocurrencies have many potential benefits including faster and more efficient settlement of payments, regulatory concerns center around their use in illegal trade (drugs, hacks and thefts, illegal pornography, even murder-for-hire), potential to fund terrorism, launder money, and avoid capital controls. There is little doubt that by providing a digital and anonymous payment mechanism, cryptocurrencies such as bitcoin have facilitated the growth of online “darknet" marketplaces in which illegal goods and services are traded. The recent FBI seizure of over $\$ 4$ million worth of bitcoin from one such marketplace, the "Silk Road," provides some idea of the scale of the problem faced by regulators.

This paper seeks to quantify and characterize the illegal trade facilitated by bitcoin. In doing so, we hope to better understand the nature and scale of the "problem" facing this nascent technology. We develop new methods for identifying illegal activity in bitcoin. These methods can also be used in analyzing many other blockchains. Several recent seizures of bitcoin by law enforcement agencies (including the US FBI's seizure of the "Silk Road" marketplace), combined with the public nature of the blockchain, provide us with a unique laboratory in which to analyze the illegal ecosystem that has evolved in the bitcoin network. Although individual identities are masked by the pseudo-anonymity of a 26-35 character alpha-numeric address, the public nature of the blockchain allows us to link bitcoin

\footnotetext{
${ }^{1}$ SEC Release No. 34-79103, March 10, 2017; and https://coinmarketcap.com.

${ }^{2}$ Source: financial research firm Autonomous Next and cnbc.com.

${ }^{3}$ Bitcoin futures commenced trading on the CME (Chicago Mercantile Exchange) on December 18, 2017 and on the Chicago Board Options Exchange (CBOE) on December 10, 2017. A bitcoin futures contract on CBOE is for one bitcoin, whereas on CBOE it is five bitcoins. At a price of approximately $\$ 20,000$ per bitcoin at the time the CME bitcoin futures launched, one CME bitcoin futures contract has a notional value of around $\$ 100,000$.
} 
transactions to individual "users" (market participants) and then further identify the users that had bitcoin seized by authorities. Bitcoin seizures (combined with a few other sources) provide us with a sample of users known to be involved in illegal activity. This is the starting point for our analysis, from which we apply two different empirical approaches to go from the sample to the estimated population of illegal activity.

Our first approach exploits the trade networks of users known to be involved in illegal activity ("illegal users"). We use the bitcoin blockchain to reconstruct the complete network of transactions between market participants. We then apply a type of network cluster analysis to identify two distinct communities in the data-the legal and illegal communities. Our second approach exploits certain characteristics that distinguish between legal and illegal bitcoin users. We use these characteristics in simultaneous equation models that identify the illegal activity while accounting for the non-randomness of the sample of known illegal users. For example, we measure the extent to which individual bitcoin users take actions to conceal their identity and trading records, which predicts involvement in illegal activity.

We find that illegal activity accounts for a substantial proportion of the users and trading activity in bitcoin. For example, approximately one-quarter of all users (26\%) and close to one-half of bitcoin transactions (46\%) are associated with illegal activity. Furthermore, approximately one-fifth (23\%) of the total dollar value of transactions and approximately one-half of bitcoin holdings (49\%) through time are associated with illegal activity using our algorithms. Our estimates suggest that in April 2017, there are an estimated 27 million bitcoin market participants that use bitcoin primarily for illegal purposes. These users annually conduct around 37 million transactions, with a value of around $\$ 76$ billion, and collectively hold around $\$ 7$ billion worth of bitcoin.

To give these numbers some context, a report to the US White House Office of National Drug Control Policy estimates that drug users in the United States in 2010 spend in the order of $\$ 100$ billion annually on illicit drugs. ${ }^{4}$ Using different methods, the size of the European market for illegal drugs is estimated to be at least $€ 24$ billion per year. ${ }^{5}$ While comparisons between such estimates and ours are imprecise for a number of reasons and the illegal activity captured by our estimates is broader than just illegal drugs, they do provide a sense that the scale of the illegal activity involving bitcoin is not only meaningful as a proportion of bitcoin activity, but also in absolute dollar terms.

\footnotetext{
${ }^{4}$ The report, prepared by the RAND Corporation, estimates the user of cocaine, crack, heroin, marijuana, and methamphetamine, and is available at (www.rand.org/t/RR534). A significant share of the illegal activity involving bitcoin is likely associated with buying/selling illegal drugs online (e.g., Soska and Christin, 2015), which is what motivates the comparison with the size of the market for illegal drugs.

${ }^{5}$ The estimate is from the European Monitoring Centre for Drugs and Drug Addiction / Europol "EU Drug Markets Report" for the year 2013 (http://www.emcdda.europa.eu/attachements.cfm/att_194336_EN_TD3112366ENC.pdf).
} 
We also uncover that the use of bitcoin in illegal trade varies through time. Since 2016, the proportion of bitcoin activity associated with illegal trade has declined, although the absolute amount has continued to increase. We attribute the declining share of illegal activity to two main factors. The first is the rapid growth in mainstream and speculative interest in bitcoin, which mechanically decreases the illegal share. For example, we find that the proportion of illegal activity in bitcoin is inversely related to the Google search intensity for the keyword "bitcoin." The second factor is the emergence of alternative "shadow" cryptocurrencies that are more opaque and better at concealing a user's activity (e.g., Dash, Monero, and ZCash). We find that the emergence of such shadow cryptocurrencies is also associated with a decrease in the proportion of illegal activity in bitcoin. Despite the emergence of alternative cryptocurrencies and numerous darknet marketplace seizures by law enforcement agencies, the amount of illegal activity involving bitcoin at the end of our sample in April 2017 remains close to its all-time high.

Bitcoin users that are involved in illegal activity differ from other users in several characteristics. Illegal users tend to transact more, but in smaller transactions. They are also more likely to repeatedly transact with a given counterparty. These differences in transactional characteristics are generally consistent with the notion that while illegal users predominantly (or solely) use bitcoin as a payment system to facilitate trade in illegal goods/services, some legal users treat bitcoin as an investment or speculative asset. Despite transacting more, illegal users tend to hold less bitcoin, consistent with them facing risks of having bitcoin holdings seized by authorities.

We find several other robust predictors of involvement in illegal activity. A user is more likely to be involved in illegal activity if they trade when there are more darknet marketplaces in operation, lower combined market value of shadow coins, less mainstream interest in bitcoin as measured by Google search intensity, and immediately following darknet marketplaces seizures or scams. A user is also more likely to be involved in illegal activity if they use "tumbling" and/or "wash trades" - two trading techniques that can help conceal one's activity.

The network of bitcoin transactions between illegal users is three to four times denser than the legal user network, with users much more connected with one another through transactions. The higher density is consistent with illegal users transacting more and using bitcoin primarily as a payment system for buying/selling goods.

It is important to consider the differences between cryptocurrencies and cash. After all, cash is also largely anonymous (traceable only through serial numbers) and has therefore traditionally played an important role in facilitating crime and illegal trade (e.g., Rogoff, 2016). The key difference is that cryptocurrencies (similar to PayPal and credit cards) enable digital transactions and thus e-commerce. Arguably, the ability to make digital payments revolutionized retail and wholesale trade. Online shopping substantially impacted the structure of retailing, consumption patterns, choice, marketing, competition, 
and ultimately supply and demand. Until cryptocurrencies, such impacts were largely limited to legal goods and services due to the traceability of digital payments. Cryptocurrencies may have changed this, by combining the anonymity of cash with digitization, which enables efficient anonymous online and cross-border commerce. Cryptocurrencies therefore have the potential to cause an important structural shift in how the black market operates.

While the emergence of illegal darknet marketplaces illustrates that this shift may have commenced, it is not obvious to what extent the black market will adopt the opportunities for e-commerce and digital payments via cryptocurrencies. This is an important empirical question. Our findings illustrate the dynamics of this adoption process and suggest that eight years after the introduction of the first cryptocurrency, the black market has indeed adopted this form of electronic payment on a meaningful scale. Thus, our results suggest that cryptocurrencies are having a material impact on the way the black market for illegal goods and services operates.

Our findings have a number of further implications. Blockchain technology and the systems/protocols that can be implemented on a blockchain have the potential to revolutionize numerous industries. In shedding light on the dark side of cryptocurrencies, we hope this research will reduce some of the regulatory uncertainty about the negative consequences and risks of this innovation, facilitating more informed policy decisions that assess both the costs and benefits. In turn, we hope this enables these technologies to reach their potential. Second, our paper contributes to understanding the intrinsic value of bitcoin, highlighting that a significant component of its value as a payment system derives from its use in facilitating illegal trade. This has ethical implications for bitcoin as an investment, as well as valuation implications. Third, our paper moves the literature closer to understanding the welfare consequences of the growth in illegal online trade. A crucial piece of this puzzle is understanding whether illegal online trade simply reflects a migration of activity that would have otherwise occurred on the street, versus the alternative that by making illegal goods more accessible, convenient to buy, and less risky to buy due to anonymity, "black e-commerce" encourages growth in the aggregate black market. Our estimates contribute to understanding this issue, but further research is required to relate these estimates to trends in the offline black market to further understand the welfare consequences.

This paper also makes a methodological contribution. The techniques developed in this paper can be used in cryptocurrency surveillance in a number of ways, including monitoring trends in illegal activity, its response to regulatory interventions, and how its characteristics change through time. The methods can also be used to identify key bitcoin users (e.g., "hubs" in the illegal trade network) which, when combined with other sources of information, can be linked to specific individuals. The techniques in this paper can also be used to study other types of activity in bitcoin or other blockchains. 
Our paper contributes to a few areas of recent literature. We add to the literature on the economics of cryptocurrencies and applications of blockchain technology to securities markets by showing that one of the major uses of cryptocurrencies as a payment system is in settings where anonymity is valued (e.g., illegal trade). ${ }^{6}$ Our paper also contributes to the computer science literature that analyzes the degree of anonymity in bitcoin. ${ }^{7}$ We exploit algorithms from this literature to identify individual users in the data, and we add new methods to the literature that go beyond observing individuals, to identification of communities and estimation of populations of users. Finally, our paper is also related to studies of darknet marketplaces and the online drug trade, including papers from computer science and drug policy. ${ }^{8} \mathrm{We}$ contribute to this literature by quantifying the amount of illegal activity that involves bitcoin, rather than studying a single market (e.g., Silk Road) or indirect lower-bound measures of darknet activity such as the feedback left by buyers. Empirically, we confirm that the estimated population of illegal activity is several times larger than what can be "observed" through studying known darknet marketplaces and their customers.

The next section provides institutional details about bitcoin and the blockchain, darknet marketplaces in which illegal goods and services are bought/sold using bitcoin, and law enforcement efforts to monitor and disrupt illegal online activity. Section 2 describes the blockchain data used in this paper. Section 3 explains three approaches that we use to construct a sample of illegal activity and characterizes that sample. The sample forms the input to our empirical methods in Section 4 that quantify the total amount of illegal activity, its trends, and its characteristics. A discussion of the implications of the results and how they relate to existing studies is in Section 5, while Section 6 concludes.

\section{Institutional details}

\subsection{The structure of the bitcoin blockchain}

Bitcoin is an international currency, not associated with any country or central bank, backed only by its limited total supply and the willingness of bitcoin users to recognize its value. ${ }^{9}$ Bitcoins are "mined" (created) by solving cryptographic puzzles that deterministically increase in difficulty and once solved can be easily verified. Each solution results in a new "block" and provides the miner with the "block reward" (currently 12.5 bitcoins), which incentivizes the miner. The difficulty of the cryptographic

\footnotetext{
${ }^{6}$ See: Malinova and Park, 2016; Khapko and Zoican, 2016; Yermack, 2017; Huberman et al., 2017; Basu et al., 2018.

${ }^{7}$ See: Meiklejohn et al., 2013; Ron and Shamir, 2013; Androulaki et al., 2013; Tasca et al., 2018.

${ }^{8}$ See: Soska and Christin, 2015; Barratt et al., 2016a; Aldridge and Décary-Hétu, 2016; Van Buskirk et al., 2016.

${ }^{9}$ As of January 2017, over 16 million bitcoins had been mined out of a maximum of 21 million. This maximum limit is built into the protocol (Nakamoto, 2008).
} 
puzzles is adjusted after every 2,016 blocks (approximately 14 days) by an amount that ensures the time between blocks remains ten minutes on average.

As well as expanding the supply of bitcoin, each block confirms a collection of recent transactions (transactions since the last block). Each block also contains a reference to the last block, thereby forming a "chain", giving rise to the term "blockchain". The blockchain thus forms a complete and sequential record of all transactions and is publically available to any participant in the network.

Bitcoins are divisible to the "Satoshi", being one hundred millionth of one bitcoin (currently worth less than two hundredths of a cent). Each bitcoin holding (or parcel) is identified by an address, analogous to the serial number of a banknote. Unlike banknotes, bitcoin does not have to be held in round units (e.g., 5, 10, 50). Due to the revelation of the private key, unless a holding of bitcoin with a given address is exactly spent in a transaction, the "change" from the transaction is returned to a new address forming a new parcel of bitcoin.

A bitcoin "user" (a participant in the network) stores the addresses associated with each parcel of bitcoin that they own in a "wallet". Similar to a conventional cash wallet, a bitcoin wallet balance is the sum of the balances of all the addresses inside the wallet. While individual bitcoin addresses are designed to be anonymous, it is possible to link addresses belonging to the same wallet when more than one address is used to make a purchase.

\subsection{Darknet marketplaces and their microstructure}

The "darknet" is a network like the internet, but that can only be accessed through particular communications protocols that provide greater anonymity than the internet. The darknet contains online marketplaces, much like EBay, but with anonymous communications, which also makes these marketplaces less accessible than online stores on the internet. Darknet marketplaces are particularly popular for trading illegal goods and services because the identities of buyers and sellers are concealed. The darknet is estimated to contain approximately 30,000 domains (Lewman, 2016).

To access a darknet marketplace, a user is generally required to establish an account (usually free) at the marketplace to browse vendor products (Martin, 2014a; Van Slobbe, 2016). Similar to the way PayPal propelled EBay, the secure, decentralized, and anonymous nature of cryptocurrencies has played an important role in the success of darknet marketplaces. While bitcoin is the most widespread cryptocurrency used in such marketplaces, other currencies have occasionally been adopted, either due to their popularity (such as Ethereum) or improved anonymity (such as Monero). Despite the availability of 
alternate currencies on some marketplaces, the vast majority of transactions on the darknet are still undertaken in bitcoin. ${ }^{10}$

A user that wants to buy goods or services on a darknet marketplace must first acquire cryptocurrency (typically from an online exchange or broker) and then deposit this in an address belonging to the darknet marketplace (often termed a "hot wallet"). These funds are held in "escrow" by the marketplace. Vendor prices on darknet markets are often quoted inclusive of a marketplace fee. The escrow system also assists marketplace administrators in mediating disputes between buyers and sellers and minimizing scams in which money is collected without the intention of ever shipping any goods (Aldridge and Décary-Hétu, 2014; Christin, 2013). Funds are released when the vendor indicates the goods have been sent. In some marketplaces, the funds are held until the buyer indicates that the goods have been received. The escrow function of the darknet marketplaces sometimes leads to "exit scams", whereby a marketplace ceases operations but does not return bitcoin held in escrow. Many such scams have been perpetrated by marketplaces in the last five years, including Sheep Marketplace (2013), Pirate Market (2014), Evolution (2015), and Nucleus (2016).

The evolution of dark marketplaces allows sellers of illegal goods and services to reach global audiences (Van Buskirk et al., 2016). This internationalization of illegal trade necessitates more complex methods of communications and logistics to avoid detection. To this end, buyers placing an order with an online seller typically communicate using PGP (Pretty Good Privacy) encryption, which encodes and decodes messages using a pair of public and private keys (Cox, 2016). On some (typically more recent) marketplaces, this functionality is built into the site. Logistically, items are typically delivered by mail and the process by which this occurs has been widely documented (Christin, 2013; Van Hout and Bingham, 2013; Lavorgna, 2016; Van Slobbe, 2016). Many methods are used to minimize the chance of such deliveries being intercepted by law enforcement, including professional logos, vacuum sealed bags, posting small quantities of product, and including a (fake) return address (Christin, 2013; Basu, 2014; Tzanetakis et al., 2016). Customers are advised by marketplaces to avoid using their real name or address to minimize the risk of being caught by law enforcement agencies (Martin, 2014b).

After receiving their goods, buyers are encouraged to leave feedback about the seller, commenting on the arrival (or otherwise) of the goods, their quality, and overall service (Van Slobbe, 2016). Such feedback is paramount for developing a reputation in a marketplace that is primarily based on trust between participants, with few ramifications for "scamming" purchasers (Aldridge and Décary-Hétu, 2014; Tzanetakis et al., 2016).

\footnotetext{
${ }^{10}$ A recent estimate from a darknet marketplace operator identified bitcoin as accounting for $98 \%$ of transactions: https://www.wired.com/2017/01/monero-drug-dealers-cryptocurrency-choice-fire/.
} 
To get a sense of how a buyer navigates a darknet marketplace, Figure 1 provides screenshots from one of the first darknet marketplaces, "Silk Road". Panel A provides an example of the "Drugs" page illustrating that a wide variety of illegal drugs, weapons, and forgeries can be purchased using bitcoin. Panel B provides an example of information about individual items and sellers. Clicking on the appropriate headings, one can obtain further information about the items (detailed description, insurance/refund policies, available postage methods and locations, security and encryption, and so on) and about the seller (their rating from buyers, detailed feedback from buyers, history of sales, and so on). Panel C shows the interface for depositing bitcoin to Silk Road's escrow account, how to transfer bitcoins to a given seller, and how to withdraw bitcoins from escrow.

\section{$<$ Figure $1>$}

By providing an anonymous, digital method of payment, bitcoin did for darknet marketplaces what PayPal did for EBay - provide a reliable, scalable, and convenient payment mechanism. What was also required was an anonymous way of hosting and accessing those illegal marketplaces. This issue is solved through the use of The Onion Router (TOR), originally developed by the US Navy. By routing the message through several nodes, the TOR network obfuscates the path (and hence the IP address) of a message sent between two clients.

The combination of TOR for covert communications and bitcoin for covert payments has led to the proliferation of darknet marketplaces. The most well-known marketplace was the "Silk Road" started in 2011. Since its shutdown by the FBI in 2013, numerous other marketplaces have sprung up (see Table A1 in Appendix A for a list). Despite frequent shutdowns, seizures and scams, measures of darknet marketplace activity indicate steady growth in the number of market participants and products (Matthews et al., 2017). For example, one of the largest marketplaces in 2017, "AlphaBay", had over 350,000 items available for sale in categories such as drugs, weapons, malware, and illegal pornography.

\subsection{Surveillance and cryptocurrency seizures from darknet marketplaces}

Cryptocurrencies have proven effective not only in facilitating illegal trade, but also in the detection of illegal activity due to the public nature of the blockchain. Even though bitcoin has been used extensively in illegal activity, some argue that the blockchain actually makes it easier for law enforcement to detect illegal activity, despite the currency's anonymity. Koshy, Koshy, and McDaniel (2014) show that by monitoring transactions transmitted from computers to the blockchain, they are able to link individual transactions to the IP address of the sender. Meiklejohn et al. (2013) describe how tracing a bitcoin theft on the blockchain to bitcoin exchanges could be used by authorities with subpoena powers to 
potentially identify perpetrators. Yermack (2017) hypothesizes that the growing popularity of bitcoin will inevitably lead to a growing market for de-anonymizing technologies, leading to increased transparency of the users making transactions on the blockchain. In response to these pressures, supporters of the anonymity provided by cryptocurrencies are actively developing new currencies that challenge law enforcement's detection methods. Such currencies include Monero, which hides user's public keys among a group of public keys that contain the same amount (known as "Ring Signatures"), and ZCash (launched in 2016), which uses zero-knowledge proofs that hide sender, recipient, and transaction amount (Noether, 2015; Ben-Sasson et al., 2014).

Recently, law enforcement agencies have been successful in seizing bitcoin from a number of darknet marketplaces. For example, the Silk Road marketplace was raided by the FBI on October 2, 2013, seizing bitcoin from customer and supplier escrow accounts (hot wallets) and from the owner/operator, Ross William Ulbricht. After the closure of the Silk Road, law enforcement agencies successfully seized bitcoin from several other illegal sites/individuals (see Table A2 of Appendix A). Numerous darknet sites were raided and shut down in "Operation Onymous"; an international collaboration between US and European law enforcement agencies that targeted illegal darknet sites. Despite the seizures, illegal darknet marketplaces continue to operate, with many new ones created after each seizure.

The seized bitcoin from these operations allows us to identify bitcoin users (customers, suppliers, and marketplace operators) involved in illegal activity. These observations provide a starting point from which to estimate the extent of illegal activity involving bitcoin.

Law enforcement agencies use a number of strategies to detect illegal activity on the darknet, ranging from cyber-surveillance to forensic analysis. Given that detected illegal activity feeds into our identification techniques, it is important to understand law enforcement strategies. Christin (2013) and Kruithof et al. (2016) describe a number of such strategies, including: infiltrating the TOR network to determine individual IP addresses, decoding the financial infrastructure of bitcoin to identify individuals, and using traditional forensic and investigative techniques on seized packages. Law enforcement agencies monitor suspicious packages passing through the postal service. Agencies also order drugs on darknet marketplaces to investigate the return address on the package. For example, an unusual amount of outgoing mail from a large Australian drug dealer led authorities to seize over 24,000 in bitcoin, along with a wide array of drugs and cash. Investigators also sometimes pose as suppliers to gather addresses of customers, thereby revealing their identities. Finally, by conducting major seizures, agencies can create distrust in the online trade of illegal drugs among participants (Van Slobbe, 2016; Christin, 2013). Largescale initiatives such as "Operation Onymous", in which law enforcement agencies shut down several illegal marketplaces and made 17 arrests across 17 countries, can discourage illegal online activity by increasing the risk of detection (Franklin, Paxson, Perrig, and Savage, 2007). 


\section{Data and descriptive statistics}

We extract the complete record of bitcoin transactions from the public bitcoin blockchain, from the first block on January 3, 2009, to the end of April 2017. For each transaction, we collect the unique transaction hash, the transaction amount, the fee, the sender and recipient addresses, the timestamp, and the block number.

\subsection{Identifying users in transaction-level bitcoin data}

The data that make up the bitcoin blockchain reveal "addresses" (identifiers for parcels of bitcoin) but not the "users" (individuals) that control those addresses. A user typically controls several addresses. This one-to-many mapping occurs partly as a result of various activities that users employ to preserve their anonymity and partly due to transaction mechanics (e.g., when a user receives "change" in a transaction, the change is given a new address). ${ }^{11} \mathrm{We}$ map addresses to individual users with the UnionFind algorithm developed by Cormen, Leiserson, Rivest, and Stein (2001) and Ron and Shamir (2013) and used in several related papers such as Meiklejohn et al. (2013). This algorithm transforms the transaction-level data into user-level data, linking each transaction to the associated users.

The following illustrates how the Union-Find algorithm works. A transaction usually involves several addresses from one user. For example, the payer ("sender") of bitcoin might send bitcoin from multiple addresses and receive change to a new address. Because a user must control the private key of each address from which bitcoin is sent in a given transaction, in the first step of the algorithm all of the sender's addresses in a given transaction are associated with one user. Transitivity is then used to link the addresses of a user across multiple transactions. For example, suppose two separate transactions are observed; one in which bitcoin is sent from addresses A and B and another in which bitcoin is sent from addresses $\mathrm{B}$ and $\mathrm{C}$. The first transaction identifies that addresses $\mathrm{A}$ and $\mathrm{B}$ belong to one user, while the second identifies that $\mathrm{B}$ and $\mathrm{C}$ belong to one user. By transitivity, all three addresses (A, B, and C) belong to the same user.

None of the existing algorithms that cluster bitcoin addresses by user has perfect accuracy. ${ }^{12}$ The Union-Find algorithm is the most widely used approach, primarily because the errors it makes (too little clustering of addresses rather than too much clustering) are conservative in most applications (Meiklejohn et al, 2013). The Union-Find algorithm might fail to cluster together two sets of addresses controlled by one user if that user never makes a transaction that uses an address from both sets. In such instances, two

\footnotetext{
${ }^{11}$ For example, individuals can send bitcoin to a "tumbling" service which then returns the bitcoin (minus a fee) to a new address, or by sending bitcoin to oneself using a newly generated address as the recipient of the transaction (Ron and Shamir, 2013).

${ }^{12}$ For example, Androulaki et al. (2013) examine two approaches using simulations and find that many, but not all, users can be correctly identified by clustering algorithms even when they try to enhance privacy by creating new addresses.
} 
or more address clusters might in fact correspond to one user. ${ }^{13}$ In contrast, the Union-Find algorithm (unlike other approaches such as those that exploit the change from transactions) is very unlikely to make the opposite and more severe error of incorrectly clustering together sets of addresses that involve more than one user. The Union-Find algorithm is a suitable choice in our application because too little clustering (and thus having instances where two or more clusters correspond to one actual user) is unlikely to have severe consequences for our empirical methods, whereas incorrectly joining multiple users into a single cluster would be far more problematic. ${ }^{14}$

The Union-Find algorithm's tendency to join too few addresses together into clusters adds bias to some of the measures in this paper. First, the sample of known illegal users, which is the starting point for our empirical analysis, will not contain all of the addresses controlled by those users. We therefore start with a smaller sample than would be the case if the clustering algorithm had 100\% accuracy. Second, measures of the number of users will be upward biased because in some cases, two or more of the clusters identified by the Union-Find algorithm will in fact be controlled by one real user. Consequently, our estimates of the total number of bitcoin users, the number of illegal users, and the number of legal users are all likely upward biased. For similar reasons, measures such as the number of transactions per user or holdings per user are likely downward biased. This bias is less of an issue when we quantify users as percentages of the total number of users. For example, our estimates of the percentage of users that are involved in illegal activity will be less biased than the absolute number of users. This bias is even less of an issue when we quantify the number of transactions, volume, or holdings of various groups of users because these measures do not rely on knowing the number of users in each group.

\subsection{Filters and data transformations}

Our blockchain dataset consists of 465,093 blocks containing 219.6 million bitcoin transactions (unique transaction hashes). In the raw blockchain data, one transaction can have several recipients. For example, in a single transaction, Alice could send five bitcoins to Bob, two bitcoins to Charlie, and 0.1 bitcoins to the miner of the block as a transaction fee. We split these raw "compound" transactions up into their components such that each transaction has only one sender and one receiver. In the previous example, Alice's compound transaction would become three separate transactions: one with Bob, one with Charlie, and one being a transaction fee sent to the block miner. Among other things, this allows us to separate transaction fees and block rewards from other transactions. After splitting compound transactions into their components, we have 815.4 million transactions.

\footnotetext{
${ }^{13}$ Meiklejohn et al. (2013) empirically find that this error is "not too common" in bitcoin blockchain analysis.

${ }^{14}$ For example, if a single user appears in the data as two or more clusters, all of those clusters could be correctly classified with the user's actual type (illegal or legal), whereas if a legal and illegal user are incorrectly clustered together, there is no way to assign a correct classification to the cluster.
} 
In this study, we are primarily interested in quantifying the amount of illegal trade that uses bitcoin. We therefore remove transaction fees and block rewards from the sample to avoid distorting the transaction counts. This step removes 208.3 million transactions. We also remove currency conversion transactions (conversions between bitcoin and fiat currency or other cryptocurrencies), by removing bitcoin exchanges and their 88.4 million transactions. These transactions do not involve trade in the sense of buying or selling goods or services and would therefore inflate our measures of transaction activity. ${ }^{15}$ For similar reasons, we remove 71.1 million transactions that reflect the "change" given back to a user in a given transaction. These transactions are akin to paying for a $\$ 30$ product with a $\$ 50$ bill and receiving $\$ 20$ of change back (our processed dataset would record this scenario as one \$30 transaction rather than two transactions). ${ }^{16}$

We also exclude transactions that have a value of less than $\$ 1$ on the day of the transaction. Such transactions reflect negligible transfers of value and are therefore used for purposes such as messages, test transactions, and tips. ${ }^{17}$ Failure to exclude these transactions could significantly skew our data, particularly measures of the proportion of transactions.

After applying these filters, we are left with 302.8 million transactions, each having one sender and one receiver. Throughout much of the paper we consider user-level statistics such as the number of transactions per user. Such measures naturally use double-counted volume as both the sender and receiver sides of each transaction are counted. Using double-counted volume, our transaction count is doubled to 605.7 million bitcoin transactions.

\subsection{Descriptive statistics of user-level variables}

Our sample has a total of approximately 106 million bitcoin users, who collectively conduct approximately 606 million transactions, transferring around $\$ 1.9$ trillion. For each user, we calculate a collection of variables that characterize features of their bitcoin transaction activity (e.g., transaction count, transaction size, transaction frequency, and number of counterparties). We also calculate a range of

\footnotetext{
${ }^{15}$ The exchanges and miners are identified via "Wallet Explorer." Wallet Explorer joins transactions into "wallets" (the equivalent of our "users") using a similar procedure to the one described above and then classifies wallets by type either on the basis of (i) having observed an address being advertised as part of a given entity (e.g., a known address from a bitcoin exchange), or (ii) having identified an entity's wallet by sending a small amount of bitcoin to the entity, where that address is linked to the larger wallet of the entity (similar to Meiklejohn et al., 2013). See https://www.walletexplorer.com.

${ }^{16}$ The bitcoin protocol forces a user to spend the entire balance of a bitcoin address when the address is used in a transaction. Therefore, when a user has say 50 bitcoins in address $\mathrm{A}$ and wants to send another user say 30 bitcoins, the compound transaction would have two components: one transaction sending 30 bitcoins from address $\mathrm{A}$ to the other user and another transaction sending the remaining 20 bitcoins from address A to a new address B that is controlled by the sending user. The latter of the two transactions is the "change" and is removed from our sample.

${ }^{17}$ These 144.7 million small transactions represent $17.8 \%$ of transactions, but less than $0.0001 \%$ of total bitcoin volume.
} 
user-level variables that are more specific indicators of the nature of the activity in which a user is likely to be engaged, such as the number of illegal darknet marketplaces that operate at the time the user transacts, the extent to which the user engages in transactions designed to conceal their activity, and the degree of interest in bitcoin at the time the user transacts (using Google search intensity). The detailed definitions of these variables are reported in Table 1.

\section{$<$ Table $1>$}

Table 2 reports descriptive statistics about the user-level variables. Focusing on the variables that characterize a user's bitcoin transaction activity (Panel A), we see that a typical (median) user engages in three bitcoin transactions (mean Transaction Count is 5.7 transactions) with three different counterparties (mean of Counterparties is around 4.2). Thus, a typical user has a low degree of concentration in counterparties, in that they do not repeatedly transact with the same counterparty (our measure of Concentration, which is a normalized Herfindahl-Hirschman Index, has a median of zero). There are a small number of highly active entities, with the most active having almost 11.4 million transactions and 4.4 million counterparties.

The average transaction size is around $\$ 5,000$, but a typical transaction (the median Transaction Size) is much smaller at $\$ 112$. Some transactions are very large, with the largest exceeding $\$ 90$ million. For most users, their first and last bitcoin transaction occurs within the same month (the median Existence Time is one month), although some users are present for many years (the maximum Existence Time is 101 months, or just over eight years). The other variables (Panel B) are more specific indicators of the nature of the activity in which a user is likely to be engaged and are thus important in our empirical models. We therefore define and discuss these variables when we turn to the empirical models.

$<$ Table $2>$

\section{Identifying a sample of illegal users}

We identify a sample of addresses (and therefore users) involved in illegal activity using three approaches described below.

\subsection{First approach: Bitcoin seizures by law enforcement agencies}

Our first approach exploits bitcoin seizures by law enforcement agencies such as the US FBI. We manually identify bitcoin seizures from news articles (via searches using Factiva) and US court records (via searches of the digital PACER records). Table A2 in Appendix A reports the list of seizures that we 
use. For each seizure, we extract information from court records and law enforcement agency disclosures about any identified bitcoin addresses or transactions (amounts and dates). From these details we uniquely identify the users involved in the illegal activity, by matching up the bitcoin address or transaction identifier with our user-level data constructed from the bitcoin blockchain.

In some cases (e.g., the US FBI's seizure of Silk Road and Ross Ulbricht's holdings, and the Australian law enforcement's seizure of Richard Pollard's holdings) the law enforcement agency auctioned the seized bitcoin to the public. Selling seized assets that are not themselves illegal is common practice among law enforcement agencies. Given the public nature of the auctions, we are able to identify the auction transactions on the bitcoin blockchain and work backwards to identify the seized bitcoin addresses, which in turn identify those individuals that were involved in illegal activity and had some or all of their bitcoin holdings seized by law enforcement agencies. Using this approach we identify 1,016 known illegal users, which we refer to as "Seized Users".

\subsection{Second approach: Illegal darknet marketplaces and their users}

Our second approach exploits the known "hot wallets" of major illegal darknet marketplaces. These are central accounts, many of which operate like escrow accounts, into which users of darknet marketplaces deposit or withdraw funds. We are able to identify 17 such marketplaces using data from the Wallet Explorer service, which in turn identifies these marketplaces using an approach similar to Meiklejohn et al. (2013), i.e., on the basis of small "probing" transactions undertaken with a given entity.

From these hot wallets, we identify slightly over six million darknet marketplace users as individuals that send to and/or receive bitcoin from a known darknet marketplace. We refer to the darknet marketplace hot wallets and their contributors/recipients as "Black Market Users".

An underlying assumption is that the trade that occurs in darknet marketplaces is illegal. This assumption is supported by ample anecdotal evidence, objective empirical evidence in the form of darknet market scrapes that show the goods and services traded there (e.g., Christin, 2013; Aldridge Décary-Hétu, 2014; Van Buskirk et al., 2014; Soska and Christin, 2015), as well as actions by law enforcement agencies, including indiscriminate seizures of all bitcoin from such markets.

\subsection{Third approach: Users identified in darknet forums}

Our third approach exploits information contained in the darknet, in particular the bitcoin addresses of users identified in darknet forums as selling goods/services. We use systematic scrapes of darknet forums from 2013 to $2017 .{ }^{18}$ This allows us to identify users that might never have been caught

\footnotetext{
${ }^{18}$ A list of known darknet markets is in Table A1 of Appendix. An archive of darknet forums during 2013-2015 is available at https://www.gwern.net/index. We scrape information from active darknet sites during 2016-2017.
} 
by authorities and might not be otherwise identified in the data through transactions with known darknet marketplaces. Users often post bitcoin addresses in cases such as fraud (they did not receive their goods), quality checking, and for the purposes of advertising the address to which funds should be sent, including in privately negotiated trade. While other studies have also scraped darknet marketplaces for certain types of information (e.g., Soska and Christin, 2015; Van Buskirk et al., 2016), as far as we know no other study has used scrapes to identify the bitcoin addresses of illegal users.

Using this approach, we identify an additional 448 users that were not already identified in either of the previous two approaches. We refer to these as "Forum Users".

\subsection{The sample of illegal users}

Table 3 shows the number of illegal users identified using the three approaches above and various measures of their activity. Together, there are 6,223,359 "observed" illegal users, representing 5.86\% of all bitcoin participants. They account for an even larger share of transactions-a total of 196 million transactions, or around one-third of all transactions (32.38\%). They also account for an even larger share of bitcoin holdings - throughout the sample period, the average dollar value of the bitcoin holdings of observed illegal users is around $\$ 1.3$ billion, which is close to half (45.28\%) of the average dollar value of holdings for all users. ${ }^{19}$ Observed illegal users control around one-quarter (26.33\%) of all bitcoin addresses and account for approximately $12.96 \%$ of the total dollar value of all bitcoin transactions.

Within the three subgroups of illegal users, the largest group in terms of number of users is the "Black market users", followed by "Seized users" and then "Forum users". Seized users and Forum users are nevertheless meaningful subgroups in terms of their share of total transactions.

$<$ Table $3>$

The results in Table 3 indicate that the sample of "observed" illegal users is already a substantial proportion of users and bitcoin transaction activity, without yet having applied methods to estimate the population of illegal users/activity. Capturing a relatively large sample of illegal activity is important because it provides rich information to our empirical methods that estimate the totality of illegal activity. The fact that the sample of illegal activity is drawn from three different approaches is also likely to help the subsequent empirical models by providing a more diverse sample.

A limitation of the sample of observed illegal users is that it predominantly contains users that are involved in buying and selling illegal goods and services online in darknet marketplaces. There are other 19 The average holdings numbers are considerably lower than current holdings because for the first few years of
bitcoin's existence, its market capitalization was much lower than it is currently. 
forms of illegal activity that involve bitcoin, such as money laundering, evasion of capital controls, payments in ransomware attacks, and bitcoin thefts. Without an initial sample of these forms of illegal activity, our empirical models are likely to underestimate their prevalence. Illegal activity that is similar in characteristics to illegal activity in darknet markets, and illegal activity that involves transacting with darknet market participants will be captured by our estimates of illegal activity, even if it is not in our sample of directly observed illegal activity. However, illegal activity that is dissimilar to darknet market activity and does not interact with such participants is unlikely to be captured by our empirical models. Thus, our estimates are likely to underestimate some forms of illegal activity involving bitcoin.

Given the nature of illegal activity could change through time, it is also important that our sample of observed illegal users spans different time periods and is not completely concentrated at one point in time. Figure 2 indicates that this is the case for our sample of observed illegal users and their activity.

These time-series show that the observed illegal users are present during all points in time throughout our sample period. Their share of activity is highest at the start of the sample in 2009, and then again during a period from 2012 to the end of 2015. The first of these periods (the year 2009) is not particularly economically meaningful as the first year or two of bitcoin's existence involves a very small number of users and transactions compared to subsequent years. In contrast, the activity in the second period, 2012-2015, is meaningful. This period corresponds to the time when illegal darknet marketplaces grew rapidly in number and popularity. Silk Road 1 was established in January 2011 and soon became a popular venue in which to buy and sell illegal goods and services (e.g., Soska and Christin, 2015). After Silk Road 1 was shut down by the US FBI in October of 2013, a large number of other illegal darknet marketplaces commenced operating throughout 2013-2015 (see Table A1 of Appendix A). Thus, perhaps somewhat unsurprisingly, the peak activity of our sample of observed illegal users coincides with substantial darknet marketplace activity. However, we also observe a reasonable number of illegal users and illegal activity outside of this peak window.

\section{$<$ Figure $2>$}

\section{Quantifying and characterizing all illegal activity}

Having identified a substantial sample of bitcoin users that are involved in illegal activity, our next step is to use the information in this sample to estimate the totality of illegal activity that uses bitcoin. We use two different methods to classify users into those that are primarily involved in illegal activity ("illegal users") and those that are primarily involved in legal activity ("legal users"). Subsequently, we measure the size and activity of the two groups. 
At an intuitive level, the first method exploits the network topology - the information about who trades with whom. Trade networks reveal "communities" of users and can thereby identify other illegal users that were not part of our initial sample. In contrast, the second method exploits characteristics that distinguish illegal users from legal users (controlling for non-random detection).

Both methods allow a user that was initially classified as an "observed" illegal user to be reclassified as a user that is predominantly engaged in legal activity (a "legal user"). Averaging across the three categories of observed illegal users, $0.17 \%$ of all users (2.84\% of observed illegal users) are reclassified as legal users by the models that exploit the network topology and $0.60 \%$ of all users $(10.17 \%$ of observed illegal users) are reclassified as legal users by the models that exploit characteristics of users. The reclassified users reflect both (i) errors in the classification models, and (ii) users that predominantly engage in legal activity but have some involvement in illegal activity. The relatively low reclassification rates suggest that most of the "observed" illegal users predominantly use bitcoin for illegal activity.

The two methods provide independent estimates of the illegal activity and its characteristics. Given that the methods rely on completely different assumptions and exploit different information, their concurrent use provides robustness and the ability to cross-validate results. The methods are described below in separate subsections. We then report the results of how many users and how much trade is estimated to be associated with illegal activity, after which we characterize the nature of the illegal users and their trading activity compared to legal users.

\subsection{Method 1: Network cluster analysis}

The first method exploits network topology to identify "communities" of users based on the transactions between users. In simple terms, the method works as follows. If users A, B, and C are known to be involved in illegal activity (e.g., their bitcoin was seized by law enforcement agencies), a user X that trades exclusively or predominantly with users A, B, or C is likely to also be involved in illegal activity. Similarly, a user Y that trades predominantly with users that are not identified as illegal is likely to be a legal user. This intuition drives the classification of users into legal and illegal on the basis of their transaction partners.

More formally, the method we apply is a network cluster analysis algorithm that takes as inputs the set of users ("nodes" in network terminology) and the trades between users ("edges" or "links" in network terminology). The output of the algorithm is an assignment of users to communities such that the "modularity" of the communities (density of links within communities and sparsity of links between communities) is maximized. The method labels a user as illegal (legal) if the disproportionate share of their transactions is with members of the illegal (legal) community. The method does not assume that 
users only engage in either legal or illegal activity-users can do both. Therefore, there will be some trades between the legal and illegal communities.

We apply a variant of the Smart Local Moving (SLM) algorithm developed by Waltman and van Eck (2013), adapted to our specific application. The algorithm's name ("smart moving") comes from the fact that the algorithm finds the underlying community structure in the network by moving nodes from one community to another, if such a move improves the model fit. The SLM algorithm is among the leading network cluster analysis algorithms. ${ }^{20}$ Applied to our data, the algorithm is as follows:

- $\quad$ Step 1: Assign all the observed illegal users to the illegal community and all of the remaining users to the legal community.

- $\quad$ Step 2: Loop through each user, performing the following action on each:

$\circ$ If the user disproportionately transacts with members of the user's currently assigned community, then leave the user in that community ${ }^{21}$;

- Otherwise, move the user to the other community (if the user is assigned to the illegal community, move the user to legal community, and vice versa).

- $\quad$ Step 3: Repeat Step 2 until, in a complete loop through all users, no user switches between communities. At that point the assignment to communities is stable and ensures that each member trades disproportionately with other members of the same community.

Due to the iterative nature of the algorithm, not all of the "observed" illegal users will necessarily remain in the illegal community. For example, it is possible that some of the users that had bitcoin seized by authorities were involved in some illegal activity (hence getting bitcoin seized) but were mainly using bitcoin for legal purposes. This will be recognized by the algorithm in Step 2 and the user will be moved to the legal community.

\subsection{Method 2: Detection-controlled estimation (DCE)}

The second method we use to estimate the population of users involved in illegal activity ("illegal users") is detection-controlled estimation (DCE). Intuitively, this method exploits the differences in the characteristics of legal and illegal users of bitcoin to probabilistically identify the population of illegal users. If we had a random sample of illegal users and a set of characteristics that differ between legal and

\footnotetext{
${ }^{20}$ For example, Emmons et al. (2016) in their comparison of multiple methods find that the SLM algorithm performs the best in terms of maximizing cluster quality metrics.

21 "Disproportionately" is if the proportion of transactions the user makes with other members of the same community is greater than or equal to the community's proportion of total transactions. In robustness tests we consider the proportion of volume transacted rather than transactions and find consistent results.
} 
illegal users (e.g., measures of the extent to which a user has employed tools to conceal their activity), this task would be relatively simple and could be achieved with standard techniques (regression, discriminant analysis, and so on). A complication is that detection (as in most settings where violators attempt to conceal their illegal activity from authorities) is not random, and this non-randomness must be accounted for to obtain unbiased estimators. ${ }^{22}$ We use "detection" in the broad sense of an illegal user having been identified by any of the three approaches to detecting illegal users described in Section 4.

Fortunately this econometric challenge is not unique to illegal activity in bitcoin and methods to overcome it exist. The same challenge occurs in quantifying other forms of misconduct such as tax evasion, fraud, insider trading, and market manipulation, as well as contexts such as nuclear power plant safety regulation breaches, cancer detection by mammograms, and so on. The standard tool for these settings is DCE. Since its development by Feinstein (1989, 1990), DCE models have been applied to various financial misconduct settings including tax evasion (Feinstein, 1991), corporate fraud (Wang et al., 2010), and market manipulation (Comerton-Forde and Putniņš, 2014). By explicitly modelling both underlying processes (violation and detection) simultaneously, one can obtain unbiased estimates of the illegal activity, which is otherwise only partially observed.

\section{$<$ Figure 3 here >}

Figure 3 illustrates the two-stage DCE model that we estimate. On the left is the starting point, the data, which in our case is the set of all bitcoin users. In the middle we have the two processes, violation (undertaking illegal activity) and detection (e.g., bitcoin seizures). On the right-hand side are the joint outcomes of those processes: the observable classifications of users into detected illegal users (the set $A$ ) and other users (the complement set $A^{C}$, comprising legal users and undetected illegal users).

The first branch models whether a bitcoin user, $i$, is predominantly involved in illegal or legal activity. This branch is modelled as an unobservable binary process $\left(L_{1 i}\right)$ driven by a continuous latent function $\left(Y_{1 i}\right)$ of a vector of characteristics, $x_{1 i}$, that can distinguish between legal and illegal users:

$$
\begin{gathered}
Y_{1 i}=\beta_{1} x_{1 i}+\epsilon_{1 i} \\
L_{1 i}=\left\{\begin{array}{ll}
1 & (\text { Illegal user }) \\
0 & (\text { Legal user })
\end{array} \text { if } \begin{array}{c}
Y_{1 i}>0 \\
Y_{1 i} \leq 0
\end{array}\right\}
\end{gathered}
$$

The second branch models whether or not an illegal user is "detected" (they enter our sample of observed illegal users). This detection process is modelled as another unobservable binary process $\left(L_{2 i}\right)$

\footnotetext{
${ }^{22}$ A further complication is that the determinants of this non-randomness are not separately observed (unlike, for example, non-respondents in a survey, or people that choose not to participate in the labor force) and therefore the classic tools to deal with sample selection bias (e.g., Heckman models) cannot be applied.
} 
driven by a different continuous latent function $\left(Y_{2 i}\right)$ of a vector of characteristics, $x_{2 i}$, that affect the probability that an illegal user is detected:

$$
\begin{gathered}
Y_{2 i}=x_{2 i} \beta_{2}+\epsilon_{2 i} \\
L_{2 i}=\left\{\begin{array}{ll}
1 & (\text { Detected }) \\
0 & (\text { Not detected })
\end{array} \text { if } \begin{array}{l}
Y_{2 i}>0 \\
Y_{2 i} \leq 0
\end{array}\right\}
\end{gathered}
$$

Both stages of the model are estimated simultaneously using maximum likelihood. The likelihood function for the model is derived in Appendix B. Intuitively, this process finds estimates for the vectors of model parameters, $\beta_{1}$ and $\beta_{2}$, that maximize the likelihood of the observed data (the classification of users into sets $A$ and $A^{C}$ ). From the estimates of $\beta_{1}$ and $\beta_{2}$, we compute each user's probability of being involved in illegal activity and construct a binary classification of legal and illegal users.

Similar to the SLM approach, the DCE model does not assume that detected illegal users were engaged solely or predominantly in illegal activity. Once the DCE model is estimated, the classification of users into legal and illegal categories can result in some detected illegal users being reclassified as predominantly legal users. ${ }^{23}$

Similar to Heckman models, identification in a DCE model without instruments is possible, relying on functional form and distributional assumptions. However, more robust identification is achieved through instrumental variables that affect one process but not the other. We take the more robust route of using instrumental variables. The next subsection describes the instrumental variables and their descriptive statistics.

\subsection{Variables used in the DCE model and their descriptive statistics}

One of the instrumental variables associated with illegal activity is the extent to which the user employs methods to conceal their identity or obfuscate their transaction history. For example, to partially conceal their identities from an observer of the bitcoin blockchain, users can use "tumbling" and "wash trades" to alter the addresses of their bitcoin holdings, increasing the difficulty of tracing their activity. Tumbling, in its simplest form, involves a user sending bitcoin to a tumbling provider who (in return for a small fee) returns the balance to a different address controlled by the user. Wash trades involve a user sending bitcoin from one address to another (new) address that they also control. Legal users have little reason to take such actions to conceal their actions (and incur associated costs). In contrast, users involved in illegal activity are likely to use these concealment techniques. As such, the use of tumbling services

\footnotetext{
${ }^{23}$ For example, suppose a user was involved in some illegal activity and had bitcoin seized by authorities but was mainly using bitcoin for legal purposes. Such a user will have characteristics that are similar to those of legal users and not very similar to illegal users, which would lead to a classification by the DCE model into the legal user category. In contrast, a predominantly illegal user, even if not detected or observed, is likely to have characteristics similar to other illegal users and therefore (after controlling for the differences in characteristics due to non-random detection) the user is likely to be classified as illegal by the DCE model.
} 
and wash trades is likely to be a predictor of whether a user is involved in illegal activity. Importantly (for this to be an instrumental variable), using wash trades and tumbling does not alter the probability of "detection" by law enforcement agencies via the seizures of bitcoin from darknet sites. The seizures confiscated all bitcoin held in darknet marketplace escrow accounts ("hot wallets") irrespective of whether the user employed tumbling or wash trades. For each user, we measure the percentage of their transactions that are tumbling or wash trades and call this variable Tumbling.

Another set of instruments for the likelihood that a user is involved in illegal activity involves time-series variables that are likely to correlate with the type of activity in which bitcoin users are engaged. For example, for each user we construct a measure of the average number of operational illegal darknet marketplaces at the time the user transacts (we label the variable Darknet Sites). All else equal, illegal transactions (and thus users involved in illegal activity) are more likely when there is a lot of illegal darknet marketplace activity than when there is little or no illegal darknet activity.

In a similar spirit, we construct a measure of the popularity of opaque cryptocurrencies (Dash, Monero, and ZCash). This measure, which we label Shadow Coins, is the average log market capitalization of the opaque cryptocurrencies at the time of a user's transaction. These "shadow coins" were developed to provide more privacy than bitcoin. If criminals are drawn to these shadow coins and start using them instead of bitcoin, the probability that a bitcoin user is involved in illegal activity will be inversely related to the market capitalization of such coins.

We also measure the popularity of bitcoin using its log market capitalization and the Google Trends search intensity for the keyword "bitcoin". We label these variables Bitcoin Market Cap and Bitcoin Hype, respectively. We also measure the popularity of other cryptocurrencies using the total $\log$ market capitalization of cryptocurrencies excluding bitcoin and the shadow coins (we label the variable Alt Coins). We measure these three variables at the time of each user's transaction and then for each user we average each variable across the user's transactions. To the extent that these variables correlate with speculative trading in bitcoin and mainstream (legal) use of cryptocurrencies, they will have an inverse association with the likelihood that a given user is involved in illegal activity. To avoid issues with colinearity, we do not concurrently include Bitcoin Market Cap and Bitcoin Hype in the DCE models.

Our final instrument for involvement in illegal activity exploits the anecdotal evidence that significant darknet marketplace shocks such as seizures of darknet marketplaces by law enforcement agencies or closures of such marketplaces due to scams or hacks result in a brief spike of transaction activity by illegal users as they turn to alternative marketplaces or relocate their holdings in response to the shock. At the same time, shocks to darknet marketplaces are unlikely to materially affect the activity of legal users. Therefore, for each user, we measure the fraction of the user's transaction value that occurs 
in the one week period after each major darknet marketplace shock (marketplace "raids", "scams", and "hacks" in Table A1 of Appendix A). We label this variable Darknet Shock Volume.

As determinants of the probability of detection, we include a binary variable for whether the user started using bitcoin (date of first bitcoin transaction) before the first bitcoin seizure by law enforcement agencies from Silk Road 1 (we label the variable Pre-Silk-Road User). Because users that enter the bitcoin network after the first seizure can only be detected in subsequent seizures, post-Silk-Road-seizure users are likely to have a lower detection probability.

A few things are worth noting about the variables used in the DCE model. First, while the instrumental variables help identify the model, they are not the only characteristics that help separate legal and illegal users - the full set of characteristics used in the model serve that purpose, including variables common to both detection and violation equations (they have different coefficients in each equation). The full list of variables is presented in Table 1. Second, identification of the model requires only one variable that is associated with either the probability of being involved in illegal activity or the probability of detection, but not both. We have more candidate instrumental variables than this minimum of one, and in robustness tests we examine how sensitive the results are to the assumptions about these instruments. We do so by relaxing the assumed exclusion restrictions on a subset of the instruments one at a time, from which we conclude that the results are not particularly sensitive to any individual instrumental variable's exclusion restriction.

Table 2 Panel B reports descriptive statistics about the variables that serve as instruments. Darknet Sites indicates that for the average bitcoin participant, there are on average 17 operational darknet marketplaces around the time of their transactions. This number ranges from a minimum of zero to a maximum of 27. Tumbling indicates that only a relatively small proportion of users (less than 25\%) engage in "tumbling" and/or "wash trades" to obscure the user's holdings. Thus, while techniques exist to help a bitcoin user conceal their activity, it appears that few bitcoin users adopt such techniques.

The variable Darknet Shock Volume indicates that while most users do not trade in the period immediately following darknet shocks (median of zero), some users conduct a large fraction of their trading during these periods, with the average bitcoin user undertaking around $17 \%$ of their trading following darknet shocks. The variable Bitcoin Hype indicates that for the average user, the intensity of Google searches for "bitcoin" is around $28 \%$ of its maximum of $100 \%$. The Pre-Silk-Road User dummy indicates that only around 7\% of all bitcoin participants started transacting before October 2013, when the first darknet marketplace seizure by law enforcement agencies occurred (the seizure of Silk Road 1 by the FBI). The mean/median and maximum of Bitcoin Market Cap are close in value indicating that the majority of users make the majority of their transactions in bitcoin around bitcoin's peak market value. 


\subsection{How much illegal activity involves bitcoin?}

Both methods - network cluster analysis (SLM) and detection-controlled estimation (DCE) arrive at probabilistic classifications of bitcoin users into those primarily involved in legal activity and those primarily involved in illegal activity. Once the users have been partitioned into the legal and illegal "communities", we use those categorizations to quantify the size and activity of the two groups.

Table 4 presents the main results at the aggregate level, across the sample period. Panel A reports the estimated size of the groups and their level of activity, while Panel B re-expresses these values as percentages for each group. First, the percentage of bitcoin users estimated to be predominantly involved in illegal activity is $29.12 \%$ using the SLM and $23.23 \%$ using the DCE, giving a midpoint estimate of about one-quarter of bitcoin users $(26.17 \%$, the average of the estimates from the two models). The $99 \%$ confidence interval around this estimate is $20.13 \%$ to $32.21 \% .{ }^{24}$ The midpoint estimate suggests around 27.81 million bitcoin users are predominantly involved in illegal activity, versus 78.44 million legal users.

The estimated number of illegal users is around four times larger than our sample of observed illegal users. Given our sample of observed illegal users is based on a comprehensive approach and includes all users that can be observed transacting with one of the known darknet marketplaces, the results suggest that without empirical methods such as the SLM or DCE, illegal activity that can be inferred from involvement with known darknet marketplaces represents only a small (and likely nonrandom) fraction of all illegal activity. Thus, our results suggest that studies of known/identifiable darknet markets (e.g., Soska and Christin, 2015; Meiklejohn et al., 2013) only scratch the surface of all illegal activity involving bitcoin.

\section{$<$ Table 4 >}

Table 4 also indicates that illegal users account for an even larger share of all transactionsaround $46.17 \%$ (45.67\% using the SLM and 46.67\% using the DCE) or approximately 280 million transactions. Thus, the average illegal user is involved in more transactions than the average legal user. This result is consistent with the notion that illegal users are likely to use bitcoin as a payment system (which involves actively transacting), whereas legal users may hold bitcoin for reasons such as speculation. A similar proportion is observed for holding values-illegal users on average hold around

\footnotetext{
${ }^{24}$ We use a form of bootstrapped standard errors to form the confidence interval. First we obtain standard errors from the DCE model using a bootstrap of 200 samples in which, for computational reasons, we are forced to reduce the sample size by taking a random sample (this is a conservative step as it inflates the estimated standard errors relative to the standard errors for the full sample size). We add to these standard errors the estimation uncertainty from the SLM model, which is captured by the users that cannot be uniquely assigned to the legal or illegal categories. We then apply the conservative bootstrapped standard errors to approximate the error in the midpoint estimate.
} 
one-half (49.22\%) of all outstanding bitcoins. One reason for the large share of illegal user holdings (relative to their share of the number of users) is related to the calculation of this variable as a time-series average. A high fraction of illegal users early in the sample (when there are fewer bitcoin users) can generate such a result even if the holdings per user are lower among illegal users compared to legal users.

Illegal users are estimated to control around $39.31 \%$ of bitcoin addresses and account for about one-fifth $(23.06 \%)$ of the dollar volume of bitcoin transactions. In dollar terms, illegal users conduct approximately $\$ 429$ billion worth of bitcoin transactions. Because illegal users account for a larger share of transactions than their share of dollar volume, they tend to make smaller value transactions than legal users. This result is consistent with illegal users primarily using bitcoin as a payment system rather than holding it as an investment or speculative asset.

Three general conclusions can be drawn from the results in Table 4. First, illegal users account for a sizeable proportion of both users and trading activity in bitcoin, with the exact proportion varying across different measures of activity and the two estimation models. Second, the estimates from both the SLM and DCE are fairly similar across the various activity measures, despite relying on completely different assumptions and information. Third, even a fairly comprehensive approach to identifying illegal activity directly (such as the approach used in the previous section and that used in other darknet market studies) only captures a small fraction of the total illegal activity, highlighting the importance of extrapolation beyond a directly observed sample.

\subsection{How does the illegal activity vary through time?}

There is interesting time-series variation in the amount of illegal activity and its share of all bitcoin activity. Figures 4 to 7 plot the estimated amount of illegal activity that uses bitcoin through time from the first block in 2009 to 2017. The figures show the estimated number of illegal users, the number and dollar value of their transactions, and the value of their bitcoin holdings. Panel B of each of the figures shows these activity measures as a percentage of the total across all bitcoin participants. ${ }^{25}$

\section{$<$ Figure 4 here > \\ $<$ Figure 5 here $>$ \\ $<$ Figure 6 here > \\ $<$ Figure 7 here >}

\footnotetext{
${ }^{25}$ Figures 4-7 use the average of the SLM and DCE model estimates. The SLM and DCE time-series estimates are separately reported in Figures A1-A8 of the Online Appendix.
} 
A pattern that is observed across all activity measures is that illegal activity, as a percentage of total bitcoin activity, tends to be high at the start of the sample in 2009, and then again from 2011 to the beginning of 2016, after which it steadily declines through to 2017 . The activity levels indicate that there is only a very small (negligible) level of activity in bitcoin until about the middle of 2011, so the activity at the start of the sample is not economically meaningful. In contrast, the high relative level of illegal activity between 2012 and 2016 is noteworthy and coincides with the growth in the number of illegal darknet marketplaces, starting with the Silk Road in 2011. After the Silk Road was shut down in October 2013, a large number of other illegal darknet marketplaces commenced operating between 2013 and 2015 (Table A1 of Appendix A).

What could drive the decline in the relative level of illegal activity from beginning of 2016 onwards? The first thing to note is that the decline is observed in relative terms (that is, illegal activity as a fraction of total bitcoin activity), but not in absolute terms. Thus, it is not the case that the level of illegal activity in bitcoin has declined in recent years; rather, there has been a disproportionate increase in the legal use of bitcoin since the beginning of 2016. For example, from the beginning of 2016 to April 2017, the estimated number of illegal bitcoin users increases from around 21 million to around 27 million, reflecting growth of almost $30 \%$, whereas the estimated number of legal bitcoin users increases from around 21 million to around 77 million, reflecting growth of around $250 \%$. The rapid growth of legal use is likely driven by factors such as increased interest from investors and speculators (e.g., the emergence of "cryptofunds", and more recently bitcoin futures) and increased mainstream adoption as a payment system (e.g., cafes and internet merchants accepting bitcoin).

The emergence of new cryptocurrencies that are better at concealing a user's activity might also have contributed to the decline in the share of illegal activity in bitcoin as criminals migrate to these more opaque alternatives. We shed some light on this issue by examining how the estimated illegal activity in bitcoin was impacted by one of the major darknet marketplaces, Alphabay, beginning to accept an opaque alternative cryptocurrency, Monero, on its platform from August 22, 2016. Given that Alphabay's adoption of Monero is only expected to impact illegal activity, we isolate the impact using a differencein-differences model of illegal and legal transaction activity in bitcoin during the eight weeks either side of August 22, 2016. The results (reported in Table A3 of the Online Appendix) show a significant decrease in the illegal activity in bitcoin after the event. Illegal users are estimated to make around 90 thousand fewer transactions in bitcoin per day after Alphabay's adoption of Monero (relative to legal users). This is an economically meaningful change given that illegal users made around 217 thousand transactions per day before the change. Figure A9 of the Online Appendix illustrates that the change in illegal activity occurs quickly around Alphabay's adoption of Monero. It also illustrates that the parallel trends assumption appears valid (further testing of this assumption could use a distributed lags approach). 
While the effect of Alphabay's adoption of Monero appears quite large, there are three further considerations when interpreting the effect size. First, Alphabay is only one of many darknet sites, and it is likely other sites began accepting Monero at a similar time. Second, it is possible that the transactions that migrate to Monero are smaller, leaving a proportionally larger dollar value of darknet activity in bitcoin. Finally, it is possible that some of the darknet participants that initially switched to Monero returned to bitcoin some time later, potentially due to the continued widespread use of bitcoin in darknet marketplaces.

The time-series of legal and illegal activity levels show strong growth in both illegal and legal activity throughout the sample period, in particular since 2012. Interestingly, the strong growth in illegal activity precedes the strong growth in legal activity — by about three or four years. Thus it seems illegal users were relatively early adopters of bitcoin as a payment system. Because of the rapid growth in the legal use of bitcoin in the final two years of the sample, the aggregate proportion of illegal bitcoin activity reported in the previous subsection understates the proportion that exists throughout most of the sample period. For example, for most of the period from 2009 to 2017, the estimated proportion of illegal users is closer to one-half than one-quarter (the aggregate estimate). The aggregate estimate is heavily influenced by the large number of legal users that enter in the last two years of the sample. Similarly, for much of the sample period, the estimated proportion of bitcoin transactions involved in illegal activity is between $60 \%$ and $80 \%$, contrasting with the aggregate estimate of around $46 \%$.

The most recent estimates of illegal activity (at the end of our sample in April 2017) suggest there are around 27 million illegal users of bitcoin. These users conduct around 37 million bitcoin transactions annually, valued at around $\$ 76$ billion, and collectively hold around $\$ 7$ billion in bitcoin. ${ }^{26}$

\subsection{What are the characteristics of illegal users?}

We assess the differences between legal and illegal user characteristics in two ways: univariate statistics that compare observed or estimated illegal users with their legal counterparts, and multivariate tests exploiting the coefficients of the estimated DCE model.

$<$ Table $5>$

Starting with a univariate difference in means, Table 5 compares the characteristics of the sample of "observed" illegal users with the characteristics of other users. The "other users" are not all legal users - they contain a mix of legal users and undetected illegal users. Therefore, the table also compares

\footnotetext{
${ }^{26}$ For these estimates, we have halved the double-counted volumes so that the estimates can be interpreted as the volume/value of goods/services bought/sold by the illegal users.
} 
the characteristics of users classified by the SLM and DCE models as being involved in illegal activity with those of the users classified as legal. Interestingly, despite being based on completely different assumptions, the SLM and DCE models generally agree on how the characteristics of legal users differ from illegal users.

The SLM and DCE models agree that illegal users tend to transact more (have a two to three times higher Transaction Count), but use smaller sized transactions (about half the average size of legal transactions). This result could be a reflection of illegal users predominantly using bitcoin to buy and sell goods and services, whereas some legal users also use bitcoin for investment and speculation. ${ }^{27}$ With the average size of an illegal transaction being around $\$ 3,000$, bitcoin transaction fees even at their peak of around $\$ 150$ (see Basu et al., 2018) are small relative to the average illegal transaction.

The models also agree that illegal users tend to hold less bitcoin (measured in dollar value) than legal users; their average Holding Value is about half that of legal users. This characteristic is consistent with the previous conjecture-legal users might tend to hold larger bitcoin balances because some use bitcoin for investment/speculation purposes, whereas for an illegal user that buys/sells illegal goods and services using bitcoin, holding a large balance is costly due to (i) opportunity costs of capital, and (ii) risks associated with having holdings seized by authorities. For these reasons, illegal users are likely to prefer holding less bitcoin and this tendency is supported by the data.

Illegal users tend to have more counterparties in total, reflecting their larger number of transactions, but tend to have a higher counterparty concentration. This suggests that illegal users are more likely to repeatedly transact with a given counterparty. This characteristic might be a reflection of illegal users repeatedly transacting with a given illegal darknet marketplace or other illegal user once trust is established from a successful initial exchange. Illegal users have a longer Existence Time (time between their first and last transactions in bitcoin), consistent with our observations from the time-series that illegal users tend to become involved in bitcoin earlier than legal users. Similarly, the differences in means also show that there is a higher proportion of Pre-Silk-Road users among the illegal users than the legal users (as indicated by the variable Pre-Silk-Road User).

The more specific indicators of illegal activity also show significant differences between the two groups. Illegal users tend to be more active during periods in which there are many illegal darknet marketplaces operating (a higher mean for the variable Darknet Sites). They make greater use of tumbling and wash trades to conceal their activity (two to three times more Tumbling). On average, a larger proportion of illegal volume, compared to legal volume, is transacted immediately following shocks to

\footnotetext{
${ }^{27}$ While the result could also reflect illegal users engaging in techniques to conceal their trading, this is less likely to be an explanation because a similar result holds in multivariate (DCE) tests that control for tumbling and wash trades.
} 
darknet marketplaces (Darknet Shock Volume). This finding is consistent with anecdotal evidence that illegal users turn to alternative marketplaces in response to darknet marketplace seizures or scams.

Interestingly, illegal users are more likely to transact in bitcoin when there is lower combined market value of "shadow coins" consistent with such coins serving as alternatives to bitcoin in illegal transactions. This result matches anecdotal accounts of shadow coins attracting attention from the illegal community for their increased privacy and recent examples of hackers demanding ransom payments in shadow coins rather than bitcoin. The result also supports the evidence that illegal activity in bitcoin decreased after a major darknet marketplace, Alphabay, adopted one of the major shadow coins, Monero, as a form of payment in August 2016.

Another interesting result is that there tends to be relatively fewer illegal users when there is less Bitcoin Hype, measured by the Google search intensity for "bitcoin". It therefore appears that Google searches for "bitcoin" are associated with mainstream (legal) adoption of bitcoin for payments, and/or speculative/investment interest in bitcoin. Similarly, there are relatively fewer illegal users when bitcoin market capitalization is higher and when other cryptocurrencies, "Alt-Coins" (excluding the opaque shadow coins), have higher value. This finding suggests that high valuations of bitcoin and other nonshadow cryptocurrencies correspond to periods of increased legal interest in cryptocurrencies.

In summary, the comparison of transactional characteristics (number and size of transactions, holdings, and counterparties) is consistent with the notion that illegal users predominantly use bitcoin for payments, whereas legal users are more likely to treat bitcoin as an investment asset. Furthermore, legal and illegal users differ with respect to when they are most active in bitcoin, with illegal users being most active when there are more darknet marketplaces, less bitcoin hype, lower bitcoin and other non-shadow cryptocurrency market capitalizations, and immediately following shocks to darknet marketplaces. The differences in characteristics for the instrumental variables are consistent with the hypothesized differences, lending support to their use as instruments.

$<$ Table $6>$

The DCE model coefficients reported in Table 6 provide multivariate tests of how the characteristics relate to the likelihood that a user is involved in illegal activity. The results confirm most of the observations made in the simple comparison of means. The effects of all of the instrumental variables are consistent with their hypothesized effects. A user is more likely to be involved in illegal activity if they trade when: (i) there are many darknet marketplaces in operation, (ii) "shadow coins" such as Monero are not widespread (low market values), (iii) the market value of bitcoin is low, and (iv) darknet marketplaces have recently experienced seizures or scams. A user is also more likely to be 
involved in illegal activity if they use tumbling and/or wash trades, transact frequently in small sized transactions, and tend to repeatedly transact with a given counterparty. The value of other non-privacy cryptocurrencies (Alt Coins) at the time a user transacts is not statistically significant after controlling for the other variables, despite Alt Coins correlating with the likelihood of illegal activity in univariate tests. The results suggest that Bitcoin Market Cap is more closely related to the amount of mainstream and speculative interest in bitcoin and therefore Alt Coins is not a significant predictor of illegal activity after controlling for the value of bitcoin.

The marginal effects in Table 6, reported in parentheses below the coefficient estimates, provide a sense of the magnitudes of the effects and their relative importance. ${ }^{28}$ For example, the marginal effects indicate that a one standard deviation increase in the number of illegal darknet marketplaces at the time a user transacts in bitcoin increases the probability of that user being involved in illegal activity by a factor of 0.661 , or $66.1 \%$ of what their probability would otherwise be. ${ }^{29}$ The magnitudes generally show that most of the determinants of involvement in illegal activity and determinants of the detection probability are economically meaningful. In particular, the instrumental variables Darknet Sites, Shadow Coins, Bitcoin Market Cap, and Darknet Shock Volume all have strong relations with the probability that a user is involved in illegal activity.

The DCE model also sheds light on the determinants of the likelihood that an illegal user is "detected" by either of our three approaches. The main instrument, Pre-Silk-Road User has a strong relation with detection, indicating that illegal users that commence transacting in bitcoin prior to the first darknet marketplace seizure in October 2013 have a higher probability of being detected. Similarly, those users that transact in bitcoin for a longer period of time (higher Existence Time), trade more frequently (higher Transaction Frequency), or tend to trade repeatedly with a given counterparty such as a darknet marketplace (higher Concentration), have a significantly higher detection probability.

Model 2 in Table 6 adds further control variables, including Holding Value and Transaction Count, and finds that the main results do not change much in response to additional control variables. A risk of adding too many transactional control variables is co-linearity between such variables. In unreported results, we also find that the main results are robust to including a measure of bitcoin

\footnotetext{
${ }^{28}$ To make the comparisons and interpretation easier, before estimating the DCE models, we standardize all variables to have mean zero and standard deviation of one. We also log transform the right skewed variables (Transaction Frequency, Size, and Count, and Holding Value) and winsorize the variables at + - three standard deviations to reduce the influence of extreme values.

${ }^{29}$ As an example of how to interpret the marginal effect of 0.661 , if a user's illegal probability is say $20 \%$, the predicted effect of a one standard deviation increase in Darknet Sites, holding all else constant, is to increase the user's probability to $20 \% \times 1.661=33.2 \%$, an increase of $66.1 \%$ of what their probability would otherwise be.
} 
volatility. Somewhat unexpectedly, bitcoin volatility around the time a user transacts in bitcoin has a positive association with the likelihood that user is involved in illegal activity, all else equal.

\subsection{What are the characteristics of the illegal user network?}

Exploiting the fact that the bitcoin blockchain provides us with a complete record of every transaction between every pair of counterparties, we briefly explore how the trade network of illegal users differs from that of legal users. Our approach is to compute a few descriptive network metrics that capture different aspects of network topology and structure for each of the two groups or "communities" separately and then compare the values between the two communities. In mapping the networks, users form the "nodes", and transactions between users form the "edges" or "links" between nodes.

$<$ Table $7>$

Table 7 reports the results. The first metric, Density, takes the range $[0,1]$ and indicates how highly connected the users are within a community (versus how sparse the connections are between users); it is the actual number of links between users within the given community (a "link" between two users means that they have transacted with one another) divided by the total potential number of links. It shows that the illegal trade network is three to four times denser in the sense that users are much more connected to one another through transactions. This observation is consistent with the fact that illegal users tend to transact more than legal users. It is also consistent with the notion that in the illegal community, bitcoin's dominant role is likely that of a payment system in buying/selling goods, whereas in the legal community, bitcoin is also used as an investment or for speculation.

Reciprocity takes the range [0,1] and indicates the tendency for users to engage in two-way interactions; it is the number of two-way links between users within the given community (a two-way link is when two users send and receive bitcoin to and from one another) divided by the total number of links within the given community (two-way and one-way). While Reciprocity is higher among illegal users than it is among legal users, it is generally very low in both communities (1\% among legal users and 3\% among illegal users). Thus, interactions between bitcoin users are generally only one-way interactions with one counterparty receiving bitcoin from the other but not vice versa.

Entropy measures the amount of heterogeneity among users in their number of links to other members of the community. It takes its minimum value of zero when all users have the same number of 
links (same degree). ${ }^{30}$ The results suggest that illegal users are a more heterogeneous group in terms of the number of links each user has with other members of the community. A driver of that heterogeneity could be that the illegal community at one end of the spectrum has darknet marketplaces that have hundreds of thousands of links to vendors and buyers, and at the other end has individual customers of a single marketplace, potential with only the one link.

A concluding observation is that both the SLM and DCE models provide a consistent picture of how legal and illegal users differ, this time in the context of their trade networks. Again, this suggests that the two different models tend to agree about the nature of the illegal activity in bitcoin.

\subsection{Robustness tests}

We conduct a number of different robustness tests. Perhaps the most rigorous robustness test of an empirical model is to compare its results with results from a completely different model/approach that makes different assumptions and draws on different information. Throughout the paper we put our two empirical models through this test. The two models, one based on a network cluster analysis algorithm and the other on a structural latent variables model drawing on observable characteristics, provide highly consistent results. The two models tend to agree, within a reasonable margin of error, on the overall levels of illegal activity, as well as the differences between legal and illegal users in terms of characteristics and network structure.

We also subject each of the models to specific tests that vary key assumptions or modelling choices. Table 8 reports the estimated amount of illegal activity for the most notable of these tests. For the SLM, we re-estimate the model using transaction volumes as the measure of interaction between users rather than transaction counts (SLM Alternative 1). We also consider a modification of the SLM algorithm in which we impose a constraint that does not allow the sample of "observed" illegal users to be moved to the legal community (SLM Alternative 2).

For the DCE model, one set of robustness tests involves examining the sensitivity to relaxing key exclusion restrictions. For example, in the baseline model, Darknet Sites (the number of operational darknet marketplaces at the time a user transacts) is included only as a determinant of illegal activity. As a robustness test (DCE Alternative 1), we include it in both equations, allowing it to also affect the probability of detection. Darknet Sites could affect detection if the existence of many darknet marketplaces is a catalyst for increased surveillance and enforcement by law enforcement authorities. We also test sensitivity to the key exclusion restriction in the detection equation by including Pre-Silk-Road User in both equations (DCE Alternative 2), thereby allowing it to also affect the probability of illegal

${ }^{30}$ Formally, Entropy $=-\sum_{d} P(d) \log [P(d)]$, where $P(d)$ is the degree distribution (probability density of the degree for each user, where a user's degree, $d$, is the number of links the user has with other members of the same community). 
activity. Finally, we relax the restriction that tumbling does not impact the probability of detection ( $D C E$ Alternative 3).

$<$ Table $8>$

Table 8 shows that the estimated overall levels of illegal activity across the various activity measures are not overly sensitive to modifications of the baseline model, although there is some variation in individual estimates of illegal activity. For example, across the various alternative model specifications, the estimated proportion of illegal users varies from a minimum of $22.29 \%$ to a maximum of $29.12 \%$. Similarly, the estimated characteristics of illegal users are not overly sensitive to these modifications (results not reported for conciseness). The Online Appendix Table A1 reports the coefficient estimates of the three DCE models described above in which we relax key exclusion restrictions, showing that the key results are also not particularly sensitive to these modifications.

We also examine the robustness of the DCE model to the initial parameter values used in estimating the model. We initialize the model with different starting values $(-1,0,+1$, and randomly drawn starting values), and find that our results are not sensitive to the choice of starting values, suggesting convergence to a global rather than local maximum of the likelihood function.

We re-estimate the standard errors used in confidence bounds around the estimated illegal activity and significance tests. Instead of the bootstrapped standard errors that we use in the main results, we instead compute standard errors using analytic expressions. We find that the analytic standard errors are considerably smaller than the bootstrapped standard errors. This finding suggests that using bootstrapped standard errors in the main results is the more conservative of the two approaches.

Finally, the characteristics of illegal users could change through time (for example, in response to seizures by law enforcement agencies), which could lead to model mis-specification. To examine this possibility, we estimate difference-in-differences models of how illegal user characteristics change after the Silk Road seizure relative to the changes in legal user characteristics. Controlling for the changes in legal user characteristics removes potentially confounding time-series variation that is due to the evolution of the bitcoin ecosystem.

Table A2 in the Online Appendix reports the difference-in-differences results using three different definitions of illegal users: illegal users identified by the SLM model, illegal users identified by the DCE model, and the directly observed sample of known illegal users that exist before and after the Silk Road seizure (corresponding to $2 \mathrm{~B}$ and $2 \mathrm{C}$ in Table 3). The changes in most of the characteristics are not statistically distinguishable from zero. The statistically significant changes, using the directly observed illegal user group, suggest that after the Silk Road seizure illegal users tend to make fewer transactions, use smaller transactions, trade at a lower frequency, and hold smaller bitcoin balances. Such 
changes could impact the DCE model estimates and given the direction of the changes they could bias against the DCE identifying users as illegal. However, all of the estimated changes are relative to legal users and therefore some of the differences might be driven by the increase in speculative and mainstream interest in bitcoin in the later years of the sample. Most of the estimated changes in characteristics are small relative to the overall means of the characteristics. Therefore, there do not appear to be major changes in illegal user characteristics following the Silk Road seizure. Simpler models of pre-post changes in the illegal user characteristics provide qualitatively similar results, also suggesting there are no major changes in illegal user characteristics. ${ }^{31}$

\section{Discussion}

\subsection{Implications}

Blockchain technology and the systems/protocols that can be implemented on a blockchain have the potential to revolutionize numerous industries. Possible benefits to securities markets include reducing equities settlement times and costs (Malinova and Park, 2016; Khapko and Zoican, 2016), increasing ownership transparency leading to improved governance (Yermack, 2017), and providing a payments system with the network externality benefits of a monopoly but the cost discipline imposed by free market competition (Huberman et al., 2017). The technology has even broader applications beyond securities markets, from national land registries, to tracking the provenance of diamonds, decentralized decision making, peer-to-peer insurance, prediction markets, online voting, distributed cloud storage, internet domain name management, conveyancing, medical record management, and many more.

This technology, however, is encountering considerable resistance, especially from regulators. Regulators are cautious due to their limited ability to regulate cryptocurrencies and the many potential but poorly understood risks associated with these innovations. The negative exposure generated by anecdotal accounts and salient examples of illegal activity no doubt contributes to regulatory concerns and risks stunting the adoption of blockchain technology, limiting its realized benefits. In quantifying and characterizing this area of concern, we hope to reduce the uncertainty about the negative consequences of cryptocurrencies, allowing for more informed decisions by policymakers that assess both the costs and benefits. Hopefully, by shedding light on the dark side of cryptocurrencies, this research will help blockchain technologies reach their full potential.

A second contribution of this paper is the development of new approaches to identifying illegal activity in bitcoin, drawing on network cluster analysis and detection-controlled estimation techniques.

\footnotetext{
${ }^{31}$ Future work could estimate DCE models that use only the characteristics that do not change through time, examine in more detail how user characteristics respond to seizures, or estimate models using only the observed illegal users that were not part of the Silk Road seizure.
} 
These methods can be used by law enforcement authorities in surveillance activities. For example, our methods can be applied to blockchain data going forward as new blocks are created, allowing authorities to keep their finger on the pulse of illegal activity in bitcoin. Applied in this way, one could monitor trends in illegal activity such as its growth or decline, its response to various regulatory interventions such as seizures, and how its characteristics change through time. Such information could help make more effective use of scarce regulatory and enforcement resources.

During our sample period, a number of opaque cryptocurrencies such as Monero, Dash, and ZCash, also known as "privacy coins", emerged and gained some degree of adoption among illegal users. For example, some darknet marketplaces started accepting Monero for payments and our estimates suggest that such events negatively impacted the amount of illegal activity in bitcoin. While it is possible that further development of privacy coins could render our approach to detecting illegal activity less useful going forward, to date the major privacy coins have been shown to fall short of offering their users complete privacy. Using various heuristics and clustering algorithms, computer science researchers have been able to recreate user-level records and transaction activity in popular privacy coins such as Monero (Möser et al., 2018; Wijaya et al. 2018) and ZCash (Kappos et al., 2018). On the basis of such findings, privacy coins are perhaps not as private as they are intended to be. Therefore, even if illegal activity continues to migrate to popular privacy coins such as Monero and ZCash, law enforcement agencies and researchers could still use our approach applied across several cryptocurrencies, including privacy coins and non-privacy coins such as Bitcoin Cash, Litecoin, and Ethereum. It is possible that at some stage a truly private coin will be created for which it is not possible to undertake the type of analysis that is in this paper.

Another surveillance application is in identifying individuals/entities of strategic importance, for example, major suppliers of illegal goods. Combining these empirical methods with other sources of information can "de-anonymize" the nameless entities identified in the data. This might be done, for example, by tracing the activity of particular individuals to the interface of bitcoin with either fiat currency or the regulated financial sector (many exchanges and brokers that convert cryptocurrencies to fiat currencies require the personal identification of clients). The methods that we develop can also be used in analyzing many other blockchains, though at present this might be more challenging for privacy coins.

Third, our finding that a substantial amount of illegal activity is facilitated by bitcoin suggests that bitcoin has contributed to the emergence of an online black market, which raises several welfare considerations. Should policymakers be concerned that people are buying and selling illegal goods such as drugs online and using the anonymity of cryptocurrencies to make the payments? This is an important question and the answer is not obvious. If the online market for illegal goods and services merely reflects 
a migration of activity that would have otherwise occurred "on the street" to the digital world of ecommerce, the illegal online activity facilitated via bitcoin might not be bad from a welfare perspective. In fact, there are many potential benefits to having illegal drugs and other goods bought and sold online rather than on the street. For example, it might be safer and lead to reduced violence (e.g., Barratt et al., 2016a). It could also increase the quality and safety of the drugs because darknet marketplaces rely heavily on user feedback and vendor online reputation, which can give a buyer access to more information about a seller's track record and product quality than when buying drugs on the street (e.g., Soska and Christin, 2015). There is also more choice in the goods offered, which has the potential to increase consumer welfare.

However, by making illegal goods more accessible, convenient, and reducing risk (due to anonymity), the darknet might encourage more consumption of illegal goods and increase reach, rather than simply migrating existing activity from the street to the online environment (Barratt et al., 2016b). Presuming illegal goods and services have negative net welfare consequences, then bitcoin and other cryptocurrencies could decrease welfare by enabling the online black market. Such negative consequences would have to be weighed up against welfare gains that also accompany cryptocurrencies.

Therefore, while our paper does not provide a definitive answer to the question of welfare effects, it does get closer to an answer by having estimated both the trends and scale of illegal activity involving bitcoin (the most widely used cryptocurrency in darknet marketplaces). Future research might quantify the relation between drug trafficking on the street vs online (drawing on our methods or estimates) to understand to what extent we are experiencing a simple migration vs an expansion in the overall market. It might also quantify the benefits of moving to an online market and contrast them with the negative consequences of any expansion in the market as a result of it being more accessible / convenient / safe.

Our results also have implications for the intrinsic value of bitcoin. The rapid increase in the price of bitcoin in recent times has prompted much debate and divided opinions among market participants and even policymakers / central banks about whether cryptocurrency valuations are disconnected from fundamentals and whether their prices reflect a bubble. In part, the debate reflects the uncertainty about how to value cryptocurrencies and how to estimate a fundamental or intrinsic value. While we do not propose a valuation model, our results provide an input to an assessment of fundamental value in the following sense. One of the intrinsic uses of cryptocurrencies, giving them some fundamental value, is as a payment system. To make payments with bitcoin, one has to hold some bitcoin; the more widespread its use as a payment system, the greater the aggregate demand for holding bitcoin to make payments, which, given the fixed supply, implies a higher price. Our results suggest that currently, as a payment system, bitcoin is relatively widely used to facilitate trade in illegal goods and services and thus the illegal use of bitcoin is likely to be a meaningful contributor to bitcoin's fundamental value. 
This observation - that a component of bitcoin's fundamental value derives from its use in illegal trade - raises a few issues. First, an ethical investor might not be comfortable investing in a security for which a meaningful component of the fundamental value derives from illegal use. Second, changes in the demand to use bitcoin in illegal trade are likely to impact its fundamental value. For example, increased attention from law enforcement agencies or increased adoption/substitution to more opaque alternative cryptocurrencies could materially decrease the fundamental value of bitcoin. Conversely, continued growth in the online black market with continued use of bitcoin, could further increase bitcoin's fundamental value. Third, the recent price appreciation of bitcoin greatly exceeds the growth in its use in illegal activity, suggesting either a substantial change in other components of bitcoin's fundamental value or a dislocation of the bitcoin price from its fundamental value.

\subsection{Relation to other literature}

This paper contributes to three branches of literature. First, several recent papers analyze the economics of cryptocurrencies and applications of blockchain technology to securities markets (e.g., Malinova and Park, 2016; Khapko and Zoican, 2016; Yermack, 2017; Huberman et al., 2017; Basu et al., 2018). Our paper contributes to this literature by showing that one of the major uses of cryptocurrencies as a payment system is in settings in which anonymity is valued (e.g., illegal trade).

Another related, although small, branch of literature examines the degree of anonymity in bitcoin by quantifying the extent to which various algorithms can identify entities/users in bitcoin blockchain data and track their activity (e.g., Meiklejohn et al., 2013; Ron and Shamir, 2013; Androulaki et al., 2013; Tasca et al., 2018). In doing so, some of these papers also provide insights about the different types of activities that use bitcoin. Of these papers, one of the closest to ours is Meiklejohn et al. (2013), who explore the bitcoin blockchain up to April 2013, clustering addresses into entities/users and manually identifying some of those entities by physically transacting with them. They are able to identify the addresses of some miners, exchanges, gambling services, and vendors/marketplaces (including one darknet marketplace), suggesting bitcoin entities are not completely anonymous. Tasca et al. (2018) use a similar approach to explore the different types of activity in bitcoin, focusing only on the largest entities, so-called "super clusters", and within that set, only those with a known identity. Fanusie and Robinson (2018) how a sample of known illegal entities, many of which are darknet marketplaces, exchange bitcoin for other currencies or "wash"/launder their bitcoin holdings. They find that among the known illegal entities that they consider, darknet marketplaces account for most of the bitcoin exchange/laundering and that bitcoins from these illegal entities are mainly exchanged/laundered through bitcoin exchanges, bitcoin mixers/tumblers, and gambling providers. 
None of these papers attempt to categorize all of the activity in bitcoin, nor do they try and quantify or characterize the population of illegal bitcoin users, which is the focus of our paper. We exploit the lack of perfect anonymity that is documented in these studies and draw on some of the techniques from this literature to construct an initial sample of known illegal users. We add new methods to this literature, extending the empirical toolkit from making direct observations about individuals, to identification of communities and estimation of populations of users.

Yin and Vatrapu (2017) compare the performance of various supervised machine learning algorithms in classifying a sample of bitcoin users. Their analysis uses a sample of known entities, which includes some darknet marketplaces and other illicit entities. The algorithms that perform the best within their sample give widely varying estimates of the proportion of illegal users in sample, from $10.95 \%$ to 29.81\%. While the study by Yin and Vatrapu focuses on the comparison of supervised machine learning algorithms, our study aims to provide comprehensive estimates of the scale and nature of illegal activity in bitcoin. Our paper therefore differs in that it analyzes all bitcoin activity, attempts to identify as much of the observable illegal activity as possible, and characterizes the trends and characteristics of the illegal activity. ${ }^{32}$

Finally, another related branch of literature is the recent studies of darknet marketplaces and the online drug trade, including papers from computer science and drug policy. For example, Soska and Christin (2015), use a web-crawler to scrape information from darknet marketplaces during 2013-2015, collecting a variety of data. Their paper provides valuable insights into these markets, including information about the types of goods and services traded (largely drugs), the number of goods listed, a lower bound on darknet turnover using posted feedback as a proxy (they do not have data on actual transactions/sales), the number of vendors, and the qualitative aspects of how these marketplaces operate (reputation, trust, feedback). The related drug policy studies often draw on other sources of information such as surveys of drug users and contribute insights such as: (i) darknet marketplaces like the Silk Road facilitate initiation into drug use or a return to drug use after cessation (Barratt et al., 2016b) and can encourage drug use through the provision of drug samples (Ladegaard, 2018); (ii) darknet forums can promote harm minimization by providing inexperienced users with support and knowledge from vendors and more experienced users (Bancroft, 2017); (iii) darknet marketplaces tend to reduce systemic violence compared with in-person drug trading because no face-to-face contact is required (Barratt et al., 2016a; Martin, 2018; Morselli et al., 2017); (iv) about one-quarter of the drugs traded on the Silk Road occur at a

\footnotetext{
${ }^{32}$ The results in Yin and Vatrapu (2017) are difficult to compare to ours for several reasons: their paper uses a nonrandom sample of bitcoin activity, whereas we analyze all bitcoin activity, they do not specify how they filter the blockchain data, cluster addresses to form entities / users or how they identify a sample of known entities as all of these steps were performed and provided by a data provider and are not reported, and their sample period is not specified.
} 
wholesale scale, suggesting that such markets might also indirectly serve drug users "on the street" by impacting dealers (Aldridge and Décary-Hétu, 2016); and (v) there are interesting cross-country differences in the use of the darknet marketplace "Agora" (Van Buskirk et al., 2016).

We contribute to this literature by quantifying the amount of illegal activity undertaken using bitcoin. All of the illegal activity captured by the existing studies of one or several darknet marketplaces is also in our measures because one of the approaches we use to construct a sample of observed illegal activity involves measuring transactions with known darknet marketplaces. However, our estimates include much more than this activity—we use direct measures of transactions rather than a lower-bound measure such as feedback, consider all known darknet marketplaces (rather than one or a few), include two other methods of obtaining a sample of illegal activity, and most importantly, we estimate models that extrapolate from the sample of observed illegal activity to the estimated population. This yields vastly different and more comprehensive estimates. Empirically, we confirm that studies of darknet marketplaces only scratch the surface of the illegal activity involving bitcoin - the estimated population of illegal activity is several times larger than what can be "observed" through studying known darknet marketplaces. Furthermore, the studies of darknet marketplaces do not analyze how the characteristics of illegal and legal bitcoin users differ, or how recent developments such as increased mainstream interest in bitcoin and the emergence of new, more opaque cryptocurrencies impacts the use of bitcoin in illegal activity. These are further contributions of our paper.

\section{Conclusion}

As an emerging FinTech innovation, cryptocurrencies and the blockchain technology on which they are based could revolutionize many aspects of the financial system, ranging from smart contracts to settlement, interbank transfers to venture capital funds, as well as applications beyond the financial system. Like many innovations, cryptocurrencies also have their dark side. We shed light on that dark side by quantifying and characterizing their use in illegal activity.

We find that illegal activity accounts for a sizable proportion of the users and trading activity in bitcoin, as well as an economically meaningful amount in dollar terms. For example, approximately onequarter of all users and close to one-half of transactions are associated with illegal activity, equating to around 27 million market participants with illegal turnover of around $\$ 76$ billion per year in recent times. Much of this illegal activity involves trading in darknet marketplaces. There are likely to be other forms of illegal activity such as evasion of capital controls that are not fully captured by our estimates.

Illegal users of bitcoin tend to transact more, in smaller sized transactions, often repeatedly transacting with a given counterparty, and they tend hold less bitcoin. These features are consistent with their use of bitcoin as a payment system rather than for investment or speculation. Illegal users also make 
greater use of transaction techniques that obscure their activity, and their activity spikes following shocks to darknet marketplaces. The proportion of bitcoin activity associated with illegal trade declines with increasing mainstream interest and hype (bitcoin market value and Google search intensity), the emergence of more opaque alternative cryptocurrencies, and with fewer operating darknet marketplaces.

Our results have a number of implications. First, by shedding light on the dark side of cryptocurrencies, we hope this research will reduce some of the regulatory uncertainty about the negative consequences and risks of this innovation, thereby allowing more informed policy decisions that weigh up the benefits and costs. In turn, we hope this contributes to these technologies reaching their full potential.

Second, the techniques developed in this paper can be used in cryptocurrency surveillance in a number of ways. The methods can be applied going forward as new blocks are added to the blockchain, allowing authorities to keep their finger on the pulse of illegal activity and monitor its trends, its responses to regulatory interventions, and how its characteristics change through time. Such information could help make more effective use of scarce regulatory and enforcement resources. The methods can also be used to identify individuals of strategic importance in illegal networks.

Third, our paper suggests that a significant component of the intrinsic value of bitcoin as a payment system derives from its use in facilitating illegal trade. This has ethical implications for those that view bitcoin as an investment, as well as valuation implications. For example, changes in the demand to use bitcoin in illegal trade (e.g., due to law enforcement crackdowns or increased adoption of more opaque cryptocurrencies in illegal trade) are likely to impact its fundamental value.

Finally, our paper moves the literature closer to answering the important question of the welfare consequences of the growth in illegal online trade. A crucial piece of this puzzle is understanding whether online illegal trade simply reflects migration of activity that would have otherwise occurred on the street, versus the alternative that by making illegal goods more accessible, convenient to buy, and less risky due to anonymity, the move online encourages growth in the aggregate black market. Our estimates of the amount of illegal trade facilitated via bitcoin through time contribute to understanding this issue, but further research is required to relate these estimates to trends in the offline black market. 


\section{Appendix A: Darknet sites and bitcoin seizures}

Table A1: Darknet sites accepting bitcoin, current and past

This table reports the 30 known darknet marketplaces with the longest operational history. For sites that remain operational (as at May 2017), the End date column states "Operational" and thus there is no Closure reason. Days operational is the number of days the site was operational before closure. Data are sourced from www.gwern.net.

\begin{tabular}{|c|c|c|c|c|}
\hline Market & Launch date & End date & $\begin{array}{l}\text { Closure } \\
\text { reason }\end{array}$ & $\begin{array}{c}\text { Days } \\
\text { operational }\end{array}$ \\
\hline Dream & November 15, 2013 & Operational & & $>1,207$ \\
\hline Outlaw & December 29, 2013 & May 16,2017 & Hacked & 1234 \\
\hline Silk Road 1 & January 31, 2011 & October 2, 2013 & Raided & 975 \\
\hline Black Market Reloaded & June 30, 2011 & December 2, 2013 & Hacked & 886 \\
\hline AlphaBay & December 22, 2014 & July 4, 2017 & Raided & 925 \\
\hline Tochka & January 30, 2015 & Operational & & $>766$ \\
\hline Crypto Market / Diabolus & February 14, 2015 & Operational & & $>751$ \\
\hline Real Deal & April 9, 2015 & Operational & & $>697$ \\
\hline Darknet Heroes & May 27, 2015 & Operational & & $>649$ \\
\hline Agora & December 3, 2013 & September 6, 2015 & Voluntary & 642 \\
\hline Nucleus & October 24, 2014 & April 13, 2016 & Scam & 537 \\
\hline Middle Earth & June 22, 2014 & November 4, 2015 & Scam & 500 \\
\hline BlackBank & February 5, 2014 & May 18, 2015 & Scam & 467 \\
\hline Evolution & January 14, 2014 & March 14, 2015 & Scam & 424 \\
\hline Silk Road Reloaded & January 13, 2015 & February 27, 2016 & Unknown & 410 \\
\hline Anarchia & May 7, 2015 & May 9, 2016 & Unknown & 368 \\
\hline Silk Road 2 & November 6, 2013 & November 5, 2014 & Raided & 364 \\
\hline The Marketplace & November 28, 2013 & November 9, 2014 & Voluntary & 346 \\
\hline Blue Sky Market & December 3, 2013 & November 5, 2014 & Raided & 337 \\
\hline Abraxas & December 13, 2014 & November 5, 2015 & Scam & 327 \\
\hline Pandora & October 21, 2013 & August 19, 2014 & Scam & 302 \\
\hline BuyItNow & April 30, 2013 & February 17, 2014 & Voluntary & 293 \\
\hline TorBazaar & January 26, 2014 & November 5, 2014 & Raided & 283 \\
\hline Sheep & February 28, 2013 & November 29, 2013 & Scam & 274 \\
\hline Cloud-Nine & February 11, 2014 & November 5, 2014 & Raided & 267 \\
\hline Pirate Market & November 29, 2013 & August 15, 2014 & Scam & 259 \\
\hline East India Company & April 28, 2015 & January 1, 2016 & Scam & 248 \\
\hline Mr Nice Guy 2 & February 21, 2015 & October 14, 2015 & Scam & 235 \\
\hline Andromeda & April 5, 2014 & November 18, 2014 & Scam & 227 \\
\hline Topix 2 & March 25, 2014 & November 5, 2014 & Voluntary & 225 \\
\hline
\end{tabular}




\section{Table A2: Bitcoin seizures}

This table reports major bitcoin seizures, the seizing authority, the owner of the seized bitcoin, the date of the seizure, and the amount (in bitcoin) seized.

\begin{tabular}{lcccc}
\hline Seizing authority & Seized entity & Owner of seized bitcoins & Date of seizure & Bitcoin seized \\
\hline Australian Government & Individual & Richard Pollard & December 1, 2012 & 24,518 \\
US government & Individual & Matthew Luke Gillum & July 23, 2013 & 1,294 \\
ICE and HSI & Individual & Cornelius Jan Slomp & August 27, 2013 & 385,000 \\
FBI & Individual & Ross William Ulbircht & October 1, 2013 & 144,000 \\
FBI & Site & Silk Road escrow & October 2, 2013 & 29,655 \\
& & accounts (many users) & & \\
\hline
\end{tabular}




\section{Appendix B: Derivations for the DCE model}

We define $I($.$) and D($.$) to be monotonic link functions that map x_{1 i} \beta_{1}$ and $x_{2 i} \beta_{2}$ to latent probabilities of a bitcoin user being involved predominantly in illegal activity, and detection of an illegal user, respectively. ${ }^{33}$ That is,

$$
\begin{gathered}
I\left(x_{1 i} \beta_{1}\right)=\operatorname{Pr}\left(L_{1 i}=1\right) \\
D\left(x_{2 i} \beta_{2}\right)=\operatorname{Pr}\left(L_{2 i}=1 \mid L_{1 i}=1\right)
\end{gathered}
$$

Table B1 reports the probabilities of various joint outcomes (represented by cells in the table). The joint outcomes are mutually exclusive and exhaustive, so the probabilities in Table B1 sum to one.

Table B1: Two-stage DCE model probability matrix

\begin{tabular}{lcc}
\hline & \multicolumn{1}{c}{ Illegal user } & Legal user \\
\hline Detected & $I\left(x_{1 i} \beta_{1}\right) D\left(x_{2 i} \beta_{2}\right)$ & 0 \\
Not detected & $I\left(x_{1 i} \beta_{1}\right)\left[1-D\left(x_{2 i} \beta_{2}\right)\right]$ & $1-I\left(x_{1 i} \beta_{1}\right)$ \\
\hline
\end{tabular}

The log likelihood of the users that end up in the detected (seized) illegal users category $(A)$ is the $\log$ of the sum (over users in $A$ ) of the probabilities of that joint outcome:

$$
\log L_{A}=\sum_{i \in A} \log \left[I\left(x_{1 i} \beta_{1}\right) D\left(x_{2 i} \beta_{2}\right)\right]
$$

Similarly, the log likelihood of the users that end up in the other category $\left(A^{C}\right)$ is the log of the sum (over users in $A^{C}$ ) of the probabilities of that joint outcome (the probability that the user is a legal one plus the probability that an illegal user is not detected):

$$
\begin{aligned}
& \log L_{A^{C}}=\sum_{i \epsilon A^{C}} \log \left[I\left(x_{1 i} \beta_{1}\right)\left[1-D\left(x_{2 i} \beta_{2}\right)\right]+1-I\left(x_{1 i} \beta_{1}\right)\right] \\
& \log L_{A^{C}}=\sum_{i \in A^{C}} \log \left[1-I\left(x_{1 i} \beta_{1}\right) D\left(x_{2 i} \beta_{2}\right)\right]
\end{aligned}
$$

Sets $A$ and $A^{C}$ constitute the universe of all bitcoin users. Therefore the full-sample log likelihood is:

$$
\log L=\sum_{i \in A} \log \left[I\left(x_{1 i} \beta_{1}\right) D\left(x_{2 i} \beta_{2}\right)\right]+\sum_{i \in A^{c}} \log \left[1-I\left(x_{1 i} \beta_{1}\right) D\left(x_{2 i} \beta_{2}\right)\right]
$$

Maximum likelihood estimation involves selecting parameter vectors $\beta_{1}$ and $\beta_{2}$ such that the function $\log L$ is maximized.

${ }^{33}$ In our implementation, the link functions are cumulative logistic distribution functions, that is, $I\left(x_{1 i} \beta_{1}\right)=$ $\frac{1}{1+e^{-x_{1 i} \beta_{1}}}, D\left(x_{2 i} \beta_{2}\right)=\frac{1}{1+e^{-x_{2 i} \beta_{2}}}$. 


\section{References}

Aldridge, J., and D. Décary-Hétu. 2014. 'Not an Ebay for drugs': The cryptomarket 'Silk Road' as a paradigm shifting criminal innovation. Working Paper.

Aldridge, J., and D. Décary-Hétu. 2016. Hidden wholesale: The drug diffusing capacity of online drug cryptomarkets. International Journal of Drug Policy 35:7-15.

Androulaki, E., G. Karame, M. Roeschlin, T. Scherer, and S. Capkun. 2013. Evaluating user privacy in bitcoin. In Proceedings of Financial Cryptography 2013.

Bancroft, A. 2017. Responsible use to responsible harm: Illicit drug use and peer harm reduction in a darknet cryptomarket. Health, Risk and Society 19:336-50.

Barratt, M. J., J. A. Ferris, and A. R. Winstock. 2016a. Safer scoring? Cryptomarkets, social supply and drug market violence. International Journal of Drug Policy 35:24-31.

Barratt, M. J., S. Lenton, A. Maddox, and M. Allen. 2016b. 'What if you live on top of a bakery and you like cakes?'-Drug use and harm trajectories before, during and after the emergence of Silk Road. International Journal of Drug Policy 35:50-57.

Basu, G. 2014. The strategic attributes of transnational smuggling: Logistics flexibility and operational stealth in the facilitation of illicit trade. Journal of Transportation Security 7:99-113.

Basu, S., D. Easley, and M. O'Hara. 2018. From mining to markets. Journal of Financial Economics (forthcoming).

Ben-Sasson, E., A. Chiesa, C. Garman, M. Green, I. Miers, E. Tromer, and M. Virza. 2014. Zerocash: Decentralized anonymous payments from bitcoin. In 2014 IEEE Symposium on Security and Privacy.

Christin, N. 2013. Traveling the silk road: A measurement analysis of a large anonymous online marketplace. In Proceedings of the 22nd International Conference on World Wide Web.

Comerton-Forde, C., and T. J. Putniņš. 2014. Stock price manipulation: Prevalence and determinants. Review of Finance 18:23-66.

Cormen, T. H., C. E. Leiserson, R. L. Rivest, and C. Stein. 2001. Introduction to Algorithms, Cambridge: MIT Press.

Cox, J. 2016. Staying in the shadows: The use of bitcoin and encryption in cryptomarkets. In The Internet and Drug Markets, Edited by EMCDDA. 41-47, Lisbon: EMCDDA.

Emmons, S., S. Kobourov, M. Gallant, and K. Börner. 2016. Analysis of network clustering algorithms and cluster quality metrics at scale. PLOS ONE 11:1-18.

Fanusie, Y., and T. Robinson. 2018. Bitcoin laundering: An analysis of illicit flows into digital currency services. Center on Sanctions and Illicit Finance memorandum. 
Feinstein, J. S. 1989. The safety regulation of US nuclear power plants: Violations, inspections, and abnormal occurrences. Journal of Political Economy 97:115-54.

Feinstein, J. S. 1990. Detection controlled estimation. Journal of Law and Economics 33:233-76.

Feinstein, J. S. 1991. An econometric analysis of income tax evasion and its detection. RAND Journal of Economics 22:14-35.

Franklin, J., A. Perrig, V. Paxson, and S. Savage. 2007. An inquiry into the nature and causes of the wealth of internet miscreants. In Proceedings of the 14th ACM Conference on Computer and Communications Security.

Huberman, G., J. D. Leshno, and C. Moallemi. 2017. Monopoly without a monopolist: An economic analysis of the bitcoin payment system. Working Paper.

Kappos, G., H. Yousaf, M. Maller, and S. Meiklejohn. 2018. An empirical analysis of anonymity in Zcash. Working Paper.

Khapko, M., and M. A. Zoican. 2016. "Smart” settlement. Working Paper.

Koshy, P., D. Koshy, and P. McDaniel. 2014. An analysis of anonymity in bitcoin using p2p network traffic. In $18^{\text {th }}$ International Conference on Financial Cryptography and Data Security.

Kruithof, K., J. Aldridge, D. Décary-Hétu, M. Sim, E. Dujso, and S. Hoorens. 2016. Internet-facilitated drugs trade. Working Paper.

Ladegaard, I. 2018. Instantly hooked? Freebies and samples of opioids, cannabis, MDMA, and other drugs in an illicit e-commerce market. Journal of Drug Issues 48.2:226-45.

Lavorgna, A. 2016. How the use of the internet is affecting drug trafficking practices. In Internet and Drug Markets, EMCDDA Insights.

Lewman, A. 2016. Tor and links with cryptomarkets. In Internet and Drug Markets, EMCDDA Insights.

Malinova, K., and A. Park. 2016. Market design for trading with blockchain technology. Working Paper.

Martin, J. 2014a. Drugs on the Dark Net: How Cryptomarkets are Transforming the Global Trade in Illicit Drugs. Berlin: Springer.

Martin, J. 2014b. Lost on the silk road: Online drug distribution and the "cryptomarket". Criminology and Criminal Justice 14:351-67.

Martin, J. 2018. Cryptomarkets, systemic violence and the "gentrification hypothesis". Addiction 113.5: 797-98.

Matthews, A., R. Sutherland, A. Peacock, J. Van Buskirk, E. Whittaker, L. Burns, and R. Bruno. 2017. I like the old stuff better than the new stuff? Subjective experiences of new psychoactive substances. International Journal of Drug Policy 40:44-49. 
Meiklejohn, S., M. Pomarole, G. Jordan, K. Levchenko, D. McCoy, G. M. Voelker, and S. Savage. 2013. A fistful of bitcoins: Characterizing payments among men with no names. In 13th ACM Internet Measurement Conference.

Morselli, C., D. Décary-Hétu, M. Paquet-Clouston, and J. Aldridge. 2017. Conflict management in illicit drug cryptomarkets. International Criminal Justice Review 27:237-54.

Möser, M., K. Soska, E. Heilman, K. Lee, H. Heffan, S. Srivastava, K. Hogan, J. Hennessey, A. Miller, A. Narayanan, and N. Christin. 2018. An empirical analysis of traceability in the monero blockchain. In Proceedings on Privacy Enhancing Technologies 2018.

Nakamoto, S. 2008. Bitcoin: A peer-to-peer electronic cash system. Working Paper.

Noether, S. 2015. Ring signature confidential transactions for monero. In IACR Cryptology ePrint Archive, 1098.

Rogoff, K. 2016. The Curse of Cash. (Princeton, NJ: Princeton University Press).

Ron, D., and A. Shamir. 2013. Quantitative analysis of the full bitcoin transaction graph. In $17^{\text {th }}$ Financial Cryptography and Data Security International Conference.

Soska, K., and N. Christin. 2015. Measuring the longitudinal evolution of the online anonymous marketplace ecosystem. In Proceedings of the 24th USENIX Conference on Security Symposium.

Tasca, P., S. Liu, and A. Hayes. 2018. The evolution of the bitcoin economy: Extracting and analyzing the network of payment relationships. The Journal of Risk Finance 19.2:94-126.

Tzanetakis, M., G. Kamphausen, B. Werse, and R. Von Laufenberg. 2016. The transparency paradox. Building trust, resolving disputes and optimising logistics on conventional and online drugs markets. International Journal of Drug Policy 35:58-68.

Van Buskirk, J., A. Roxburgh, M. Farrell, and L. Burns. 2014. The closure of the silk road: What has this meant for online drug trading?. Addiction 109:517-18.

Van Buskirk, J., S. Naicker, A. Roxburgh, R. Bruno, and L. Burns. 2016. Who sells what? Country specific differences in substance availability on the agora cryptomarket. International Journal of Drug Policy 35:16-23.

Van Hout, M. C., and T. Bingham. 2013. 'Surfing the silk road': A study of users' experiences. International Journal of Drug Policy 24:524-29.

Van Slobbe, J. 2016. The drug trade on the deep web: A law enforcement perspective. In Internet and Drug Markets, EMCDDA Insights.

Waltman, L., and N. Jan Van Eck. 2013. A smart local moving algorithm for large-scale modularitybased community detection. The European Physical Journal B 86:1-14.

Wang, T. Y., A. Winton, and X. Yu. 2010. Corporate fraud and business conditions: Evidence from IPOs. Journal of Finance 65:2255-92. 
Wijaya, D.A., J. Liu, R. Steinfeld, and D. Liu. 2018. Monero ring attack: Recreating zero mixin transaction effect. In 2018 17th IEEE International Conference On Trust, Security And Privacy In Computing And Communications/12th IEEE International Conference On Big Data Science And Engineering (TrustCom/BigDataSE): 1196-1201.

Yermack, D. 2017. Corporate governance and blockchains. Review of Finance 21:7-31.

Yin, H. S., and R. Vatrapu. 2017. A first estimation of the proportion of cybercriminal entities in the Bitcoin ecosystem using supervised machine learning. In Proceedings of the 2017 IEEE International Conference on Big Data: 3690-99. 


\section{Table 1}

\section{Variable definitions}

This table defines the variables that we compute for each bitcoin user. The third column, DCE equation, specifies whether the variable is used in the first equation of the DCE model (the equation modelling whether the user is involved in illegal activity, $I$ ), the second equation of the DCE model (the equation modelling whether a user that is involved in illegal activity is "detected", e.g., seized by law enforcement agencies, $D)$, both equations $(I \& D)$, or as a control variable $(C)$.

\begin{tabular}{|c|c|c|}
\hline Variable & Definition & $\begin{array}{l}\text { DCE } \\
\text { equation }\end{array}$ \\
\hline $\begin{array}{l}\text { Transaction } \\
\text { Count }\end{array}$ & $\begin{array}{l}\text { The total number of bitcoin transactions involving the given user (where the user is a sender } \\
\text { and/or recipient of bitcoin). }\end{array}$ & $\mathrm{C}$ \\
\hline Transaction Size & $\begin{array}{l}\text { Average USD value of the user's transactions. The transaction size is converted from bitcoin } \\
\text { to USD using end of day USD/BTC conversion rates. Exchange rates prior to July 18, } 2010 \\
\text { are not available and therefore values before this date are converted at the July } 18,2010 \\
\text { exchange rate. }\end{array}$ & I \& D \\
\hline $\begin{array}{l}\text { Transaction } \\
\text { Frequency }\end{array}$ & $\begin{array}{l}\text { The number of bitcoin transactions made by the user per month. This is computed as } \\
\text { Transaction Count divided by Existence Time. }\end{array}$ & I \& D \\
\hline Counterparties & The total number of other users with which the given user has transacted. & $\mathrm{C}$ \\
\hline Holding Value & $\begin{array}{l}\text { The average USD value of the user's bitcoin holdings. The average is computed from the } \\
\text { holding balances recorded at the end of each of the user's bitcoin transactions. Holding values } \\
\text { are converted from bitcoin to USD using end of day USD/BTC conversion rates. Exchange } \\
\text { rates prior to July } 18,2010 \text { are not available and therefore values before this date are } \\
\text { converted at the July } 18,2010 \text { exchange rate. }\end{array}$ & $\mathrm{C}$ \\
\hline Concentration & $\begin{array}{l}\text { Concentration is a measure of the tendency for a user to transact with one or many } \\
\text { counterparties. It ranges from } 1 \text { for a highly concentrated user who transacts with only one } \\
\text { counterparty, to } 0 \text { for a user that has many transactions, each with a different counterparty. } \\
\text { Formally, it is computed using an adaptation of a normalized Herfindahl-Hirschman Index: } \\
\text { Concentration }=\left\{1-\left[\frac{\left.\left[\left(\frac{C}{T}\right)-\left(\frac{1}{T}\right)\right]\right]}{\left.1-\left(\frac{1}{T}\right)\right]} \text { if } T>1\right.\right. \\
\text { where } T \text { is Transaction Count and } C \text { is Counterparties (the total number of other users with } \\
\text { which the given user has transacted). }\end{array}$ & I \& D \\
\hline Existence Time & $\begin{array}{l}\text { Number of months the bitcoin user is active in the bitcoin network. Measured as the number } \\
\text { of months from the user's first transaction until the user's last observed transaction, if that } \\
\text { transaction results in the user having a bitcoin balance of zero. If the user's last transaction } \\
\text { results in a bitcoin balance above zero, the user is regarded as active until the end of our } \\
\text { sample in April } 2017 \text {. }\end{array}$ & $\mathrm{I} \& \mathrm{D}$ \\
\hline Darknet Sites & $\begin{array}{l}\text { A transaction-weighted average of the number of operational illegal darknet marketplaces at } \\
\text { the time a user transacts (the sum of number of operational darknet marketplaces at every } \\
\text { transaction, divided by Transaction Count). The logic is that if a user transacts at a time when } \\
\text { there is a lot of illegal darknet marketplace activity, they are more likely to be involved in } \\
\text { illegal activity than if they are active when there is little or no illegal darknet activity. }\end{array}$ & I \& D \\
\hline
\end{tabular}




\begin{tabular}{|c|c|c|}
\hline Variable & Definition & $\begin{array}{l}\text { DCE } \\
\text { equation }\end{array}$ \\
\hline Tumbling & $\begin{array}{l}\text { Tumbling refers to techniques or services used to obscure a user's holdings or transaction } \\
\text { history. Wash transactions, in which a user is both the sender and receiver of bitcoin, are also } \\
\text { sometimes used for such purpose. Illegal users are likely to have greater incentives to obscure } \\
\text { their activity than legal users. We classify tumbling transactions using the following three } \\
\text { approaches. Approach } 1 \text { : transactions with known tumbling service providers (such as Coin } \\
\text { Fog). Approach 2: transactions in which a user sends bitcoin to another user (potential } \\
\text { tumbler) and that user sends the bitcoin back (in one or several transactions), less a tumbling } \\
\text { fee of between } 0 \text { to } 10 \% \text { within } 10 \text { blocks. Approach } 3: \text { transactions with users that display } \\
\text { the characteristics of tumbling service providers (a Transaction Count of } 10 \text { or above and } \\
\text { displays the two tumbling characteristics above in at least } 8 \% \text { of transactions). For each user, } \\
\text { we compute their percentage of tumbling and wash transactions out of their total number of } \\
\text { transactions. }\end{array}$ & $\mathrm{I}$ \\
\hline $\begin{array}{l}\text { Darknet Shock } \\
\text { Volume }\end{array}$ & $\begin{array}{l}\text { The percentage of the user's transaction value that occurs immediately after shocks to darknet } \\
\text { marketplaces, including one week after each seizure or "exit scam" of a darknet marketplace. } \\
\text { Seizures by law enforcement officials and "exit scams" in which darknet sites close without } \\
\text { warning are likely to result in increased activity from illegal users as they turn to alternative } \\
\text { marketplaces or relocate their holdings. At the same time, shocks to darknet marketplaces are } \\
\text { unlikely to materially affect the activity of legal users. }\end{array}$ & I \\
\hline Bitcoin Hype & $\begin{array}{l}\text { The transaction-weighted average of the Google Trends value for "bitcoin" (calculated from } \\
\text { Jan 1, } 2009 \text { to May 1, 2017). For each user, we record the intensity of Google searches for the } \\
\text { term "bitcoin" (scaled from 0-100) in the months of their transactions and then compute the } \\
\text { average for each user across all of their transactions. The logic is that the more intensive the } \\
\text { search activity for bitcoin on Google is, the more likely the user is transacting for speculative } \\
\text { (as opposed to illegal) purposes. }\end{array}$ & $\mathrm{I}$ \\
\hline $\begin{array}{l}\text { Bitcoin Market } \\
\text { Cap }\end{array}$ & $\begin{array}{l}\text { The transaction-weighted average log market capitalization of bitcoin at the time of each } \\
\text { user's transactions. For each user, we calculate the log market capitalization of bitcoin at the } \\
\text { time of each user's transactions. We then compute the average across all of the user's } \\
\text { transactions. The logic is that as the value of bitcoin increases, the likelihood of illegal } \\
\text { activity is lower as more speculators are present. }\end{array}$ & I \\
\hline Shadow Coins & $\begin{array}{l}\text { The transaction-weighted average log market capitalization of major opaque cryptocurrencies } \\
\text { ("shadow coins": Dash, Monero, and ZCash) at the time of each user's transactions. The logic } \\
\text { is that if illegal users make use of shadow coins, the likelihood of illegal activity in bitcoin } \\
\text { will be lower when shadow coins are more prevalent. }\end{array}$ & I \\
\hline Alt Coins & $\begin{array}{l}\text { The transaction-weighted average log market capitalization of other non-privacy coins ("alt- } \\
\text { coins": all cryptocurrencies excluding bitcoin and "shadow coins") at the time of each user's } \\
\text { transactions. The logic is that when there is a lot of interest in alternative non-privacy } \\
\text { cryptocurrencies, which cannot be used in darknet markets, all else equal, it is likely there is } \\
\text { proportionally more legal/speculative trade in cryptocurrencies and thus a lower fraction of } \\
\text { illegal activity. }\end{array}$ & I \\
\hline $\begin{array}{l}\text { Pre-Silk-Road } \\
\text { User }\end{array}$ & $\begin{array}{l}\text { Dummy variable that is equal to one if the user commenced transacting in bitcoin prior to the } \\
\text { seizure of Silk Road } 1 \text { on October } 1,2013 \text {. The logic is that an illegal user that was using } \\
\text { bitcoin prior to the first major darknet seizure by law enforcement authorities has a higher } \\
\text { probability of having been detected than a user that started transacting in bitcoin after that } \\
\text { seizure because such a user could not have been "detected" in the first seizure. }\end{array}$ & $\mathrm{D}$ \\
\hline
\end{tabular}


Table 2

Descriptive statistics for all users

This table reports descriptive statistics about bitcoin users. Transaction Count is the total number of bitcoin transactions involving a given user. Transaction Size (in USD) is the user's average transaction value. Transaction Frequency is the average rate at which the user transacts between their first and last transactions, annualized to transactions per year. Counterparties is the total number of other users with which the given user has transacted. Holding Value is the average value of the user's bitcoin holdings (in USD), where holdings are measured after each transaction. Concentration takes values between zero and one, with higher values indicating a tendency to repeatedly trade with a smaller number of counterparties. Existence Time is the number of months between the date of the user's first and last transaction. Darknet Sites is the average number of operational darknet sites at the time of each of the user's transactions. Tumbling is the percentage of the user's transactions that attempt to obscure the user's holdings (wash or tumbling trades). Darknet Shock Volume is the percentage of the user's total dollar volume that is transacted during the week after marketplace seizures or "exit scams". Bitcoin Hype is a measure of the intensity of Google searches for the term "bitcoin" around the time of the user's trades. Pre-Silk-Road User is a dummy variable taking the value one if the user's first bitcoin transaction is before the seizure of the Silk Road on October 2013. Bitcoin Market Cap, Shadow Coins, and Alt Coins are transaction-weighted average log market capitalizations of bitcoin, major opaque cryptocurrencies, and non-privacy cryptocurrencies excluding bitcoin, respectively, at the time of each user's transactions.

\begin{tabular}{lccccccc}
\hline Variable & Mean & StdDev & Min & P25 & Median & P75 & Max \\
\hline Panel A: Transactional characteristics & & & & & & \\
\hline Transaction Count & 5.70 & $1,622.74$ & 1.00 & 2.00 & 3.00 & 3.00 & $11,410,691.00$ \\
Transaction Size & $5,207.61$ & $56,939.00$ & 1.00 & 22.06 & 111.91 & 668.44 & $92,504,688.00$ \\
Transaction Frequency & 29.88 & 659.27 & 0.12 & 7.20 & 24.00 & 36.00 & $3,077,978.00$ \\
Counterparties & 4.18 & 553.71 & 0.00 & 2.00 & 3.00 & 3.00 & $4,385,500.00$ \\
Holding Value & $3,974.05$ & $55,011.00$ & 0.00 & 15.91 & 83.96 & 551.37 & $115,529,839.00$ \\
Concentration & 0.10 & 0.28 & 0.00 & 0.00 & 0.00 & 0.00 & 1.00 \\
Existence Time & 6.61 & 11.91 & 1.00 & 1.00 & 1.00 & 5.00 & 101.00 \\
\hline Panel B: Characteristics associated with particular types of activity & & & \\
\hline Darknet Sites & 17.14 & 5.10 & 0.00 & 15.00 & 18.00 & 20.00 & 27.00 \\
Tumbling & 0.45 & 0.05 & 0.00 & 0.00 & 0.00 & 0.00 & 181.82 \\
Darknet Shock Volume & 16.51 & 0.36 & 0.00 & 0.00 & 0.00 & 0.00 & 100.00 \\
Bitcoin Hype & 28.29 & 15.44 & 0.00 & 19.00 & 24.00 & 38.00 & 100.00 \\
Pre-Silk-Road User & 0.07 & 0.26 & 0.00 & 0.00 & 0.00 & 0.00 & 1.00 \\
Bitcoin Market Cap & 9.82 & 0.49 & 5.14 & 9.71 & 9.94 & 10.09 & 10.40 \\
Shadow Coins & 7.07 & 2.52 & 0.00 & 7.28 & 7.78 & 8.32 & 9.10 \\
Alt Coins & 8.68 & 2.04 & 0.00 & 8.76 & 9.21 & 9.34 & 10.26 \\
\hline
\end{tabular}


Table 3

Size and activity of observed user groups

This table reports the size and activity of (1) all users, (2) observed illegal users, and (3) other users. The observed illegal user group has three subgroups: users that had bitcoin seized by law enforcement agencies ("Seized Users"), illegal darknet marketplace escrow accounts (hot wallets), users that have interacted (sent or received bitcoin) with those accounts ("Black Market Users"), and users whose bitcoin address(es) are mentioned in darknet forums ("Forum Users"). The measures of group size and activity are: the number of users (Users), the number of transactions (Transaction Count), the average dollar value of monthly bitcoin holdings (Holding Value), the number of bitcoin addresses (Number Of Addresses), and the dollar volume of transactions (Volume). The percentage of total users/activity is reported in parentheses below each value.

\begin{tabular}{lccccc}
\hline Group / Subgroup & Users & $\begin{array}{c}\text { Transaction } \\
\text { Count (Mil) }\end{array}$ & $\begin{array}{c}\text { Holding } \\
\text { Value (\$Mil) }\end{array}$ & $\begin{array}{c}\text { Number Of } \\
\text { Addresses (Mil) }\end{array}$ & $\begin{array}{c}\text { Volume } \\
(\$ \mathrm{Bil})\end{array}$ \\
\hline 1. All Users & $106,244,432$ & 605.69 & $2,964.66$ & 221.71 & $1,862.51$ \\
& $(100.00 \%)$ & $(100.00 \%)$ & $(100.00 \%)$ & $(100.00 \%)$ & $(100.00 \%)$ \\
2. Observed Illegal Users & $6,223,359$ & 196.11 & $1,342.43$ & 58.38 & 241.46 \\
& $(5.86 \%)$ & $(32.38 \%)$ & $(45.28 \%)$ & $(26.33 \%)$ & $(12.96 \%)$ \\
2A. Seized Users & 1,041 & 23.83 & 9.39 & 8.30 & 17.51 \\
& $(0.00 \%)$ & $(3.93 \%)$ & $(0.32 \%)$ & $(3.74 \%)$ & $(0.94 \%)$ \\
2B. Black Market Users & $6,221,870$ & 157.30 & $1,324.32$ & 49.71 & 220.91 \\
$\quad$ not in 2A) & $(5.86 \%)$ & $(25.97 \%)$ & $(44.67 \%)$ & $(22.42 \%)$ & $(11.86 \%)$ \\
2C. Forum Users & 448 & 14.98 & 8.72 & 0.38 & 3.03 \\
$\quad$ not in 2A or 2B) & $(0.00 \%)$ & $(2.47 \%)$ & $(0.29 \%)$ & $(0.17 \%)$ & $(0.16 \%)$ \\
3. Other Users & $100,021,073$ & 409.58 & $1,622.23$ & 163.33 & $1,621.05$ \\
& $(94.14 \%)$ & $(67.62 \%)$ & $(54.72 \%)$ & $(73.67 \%)$ & $(87.04 \%)$ \\
\hline
\end{tabular}


Table 4

Estimated size and activity of legal and illegal user groups

This table reports the size and activity of legal and illegal user groups. The measures of group size and activity are: the number of users (Users), the number of transactions (Transaction Count), the average dollar value of bitcoin holdings (Holding Value), the number of bitcoin addresses (Number Of Addresses), and the dollar volume of transactions (Volume). Panel A reports the values of these measures for the two user groups, while Panel B expresses the measures for each group as a percentage of the total. Different rows report different approaches to classifying the legal and illegal user groups. SLM provides estimates from the network cluster analysis approach to classification (a variant of the "Smart Local Moving" algorithm). DCE provides estimates from the detectioncontrolled estimation (DCE) approach to classification, which exploits the characteristics of legal and illegal users. Midpoint is the average of the estimates from the SLM and DCE models. Upper bound and Lower bound provide a 99\% confidence interval around the Midpoint, using a form of bootstrapped standard errors.

\begin{tabular}{llccccc}
\hline Group & Classification & $\begin{array}{c}\text { Users } \\
\text { (Mil) }\end{array}$ & $\begin{array}{c}\text { Transaction } \\
\text { Count (Mil) }\end{array}$ & $\begin{array}{c}\text { Holding } \\
\text { Value (\$Mil) }\end{array}$ & $\begin{array}{c}\text { Number Of } \\
\text { Addresses (Mil) }\end{array}$ & $\begin{array}{c}\text { Volume } \\
(\$ B i l)\end{array}$ \\
\hline Panel A: Values & & & & & \\
\hline Illegal & SLM & 30.94 & 276.63 & $1,394.76$ & 87.95 & 436.78 \\
& DCE & 24.68 & 282.70 & $1,523.87$ & 86.35 & 422.05 \\
& Upper bound & 34.22 & 308.72 & $1,782.44$ & 99.67 & 558.23 \\
& Midpoint & 27.81 & 279.67 & $1,459.32$ & 87.15 & 429.41 \\
& Lower bound & 21.39 & 250.62 & $1,136.20$ & 74.63 & 300.60 \\
\hline Legal & SLM & 75.31 & 329.06 & $1,569.90$ & 133.76 & $1,425.73$ \\
& DCE & 81.57 & 322.99 & $1,440.79$ & 135.37 & $1,440.45$ \\
& Upper bound & 84.86 & 355.07 & $1,828.47$ & 147.09 & $1,561.91$ \\
& Midpoint & 78.44 & 326.02 & $1,505.35$ & 134.56 & $1,433.09$ \\
& Lower bound & 72.02 & 296.97 & $1,182.22$ & 122.04 & $1,304.28$ \\
\hline Panel B: Percentages & & & & & \\
\hline Illegal & SLM & $29.12 \%$ & $45.67 \%$ & $47.05 \%$ & $39.67 \%$ & $23.45 \%$ \\
& DCE & $23.23 \%$ & $46.67 \%$ & $51.40 \%$ & $38.95 \%$ & $22.66 \%$ \\
& Upper bound & $32.21 \%$ & $50.97 \%$ & $60.12 \%$ & $44.96 \%$ & $29.97 \%$ \\
& Midpoint & $26.17 \%$ & $46.17 \%$ & $49.22 \%$ & $39.31 \%$ & $23.06 \%$ \\
& Lower bound & $20.13 \%$ & $41.38 \%$ & $38.32 \%$ & $33.66 \%$ & $16.14 \%$ \\
\hline Legal & SLM & $70.88 \%$ & $54.33 \%$ & $52.95 \%$ & $60.33 \%$ & $76.55 \%$ \\
& DCE & $76.77 \%$ & $53.33 \%$ & $48.60 \%$ & $61.05 \%$ & $77.34 \%$ \\
& Upper bound & $79.87 \%$ & $58.62 \%$ & $61.68 \%$ & $66.34 \%$ & $83.86 \%$ \\
& Midpoint & $73.83 \%$ & $53.83 \%$ & $50.78 \%$ & $60.69 \%$ & $76.94 \%$ \\
& Lower bound & $67.79 \%$ & $49.03 \%$ & $39.88 \%$ & $55.04 \%$ & $70.03 \%$ \\
\hline
\end{tabular}


Table 5

Differences in characteristics between illegal and legal users

This table reports differences in mean characteristics for illegal vs legal bitcoin users. The first three columns ("Observed") compare observed illegal users (those identified through seizures, darknet marketplaces, and darknet forums) and other users (including both legal and undetected illegal users). The second three columns ("SLM") compare illegal and legal users, classified by a network cluster analysis algorithm (SLM). The final three columns ("DCE") compare illegal and legal users, classified by a detection-controlled estimation (DCE) model. The characteristics are as follows. Transaction Count is the total number of bitcoin transactions involving the given user. Transaction Size (in USD) is the user's average transaction value. Transaction Frequency is the average rate at which the user transacts between their first and last transactions, annualized to transactions per year. Counterparties is the total number of other users with which the given user has transacted. Holding Value is the average value of the user's bitcoin holdings (in USD), where holdings are measured after each transaction. Concentration takes values between zero and one, with higher values indicating a tendency to repeatedly trade with a smaller number of counterparties. Existence Time is the number of months between the date of the user's first and last transaction. Darknet Sites is the average number of operational darknet sites at the time of each of the user's transactions. Tumbling is the percentage of the user's transactions that attempt to obscure the user's holdings (wash or tumbling trades). Darknet Shock Volume is the percentage of the user's total dollar volume that is transacted during the week after marketplace seizures or "exit scams". Bitcoin Hype is a measure of the intensity of Google searches for the term "bitcoin" around the time of the user's trades. Pre-Silk-Road User is a dummy variable taking the value one if the user's first bitcoin transaction is before the seizure of the Silk Road on October 2013. Bitcoin Market Cap, Shadow Coins, and Alt Coins are transaction-weighted average log market capitalizations of bitcoin, major opaque cryptocurrencies, and non-privacy cryptocurrencies excluding bitcoin, respectively, at the time of each user's transactions. The significance of the difference in means is computed with t-tests. $*^{* *}, * *$, and $*$ indicate statistical significance at the $1 \%, 5 \%$, and $10 \%$ levels respectively.

\begin{tabular}{|c|c|c|c|c|c|c|c|c|c|}
\hline \multirow[t]{2}{*}{ Variable } & \multicolumn{3}{|c|}{ Observed } & \multicolumn{3}{|c|}{ SLM } & \multicolumn{3}{|c|}{$\mathrm{DCE}$} \\
\hline & $\begin{array}{l}\text { Other } \\
(1)\end{array}$ & $\begin{array}{c}\text { Illegal } \\
(2)\end{array}$ & $\begin{array}{c}\text { Difference } \\
(2-1) \\
\end{array}$ & $\begin{array}{l}\text { Legal } \\
(1)\end{array}$ & $\begin{array}{l}\text { Illegal } \\
(2)\end{array}$ & $\begin{array}{c}\text { Difference } \\
(2-1)\end{array}$ & $\begin{array}{c}\text { Legal } \\
(1)\end{array}$ & $\begin{array}{l}\text { Illegal } \\
(2)\end{array}$ & $\begin{array}{c}\text { Difference } \\
(2-1)\end{array}$ \\
\hline Transaction Count & 4.09 & 31.51 & $27.42 * * *$ & 4.37 & 8.94 & $4.57 * * *$ & 3.96 & 11.46 & $7.50 * * *$ \\
\hline Transaction Size & $5,346.87$ & $2,969.38$ & $-2,377.49 * * *$ & $6,225.51$ & $2,729.66$ & $-3,495.85^{* * *}$ & $5,791.25$ & $3,278.30$ & $-2,512.95 * * *$ \\
\hline Transaction Frequency & 28.91 & 45.46 & $16.54 * * *$ & 29.77 & 30.16 & $0.39 * *$ & 28.50 & 34.45 & $5.95 * * *$ \\
\hline Counterparties & 3.53 & 14.61 & $11.08 * * *$ & 3.77 & 5.18 & $1.42 * * *$ & 3.57 & 6.19 & $2.62 * * *$ \\
\hline Holding Value & $4,021.77$ & $3,207.06$ & $-814.71 * * *$ & $4,625.45$ & $2,388.31$ & $-2,237.14 * * *$ & $4,359.86$ & $2,698.71$ & $-1,661.16^{* * *}$ \\
\hline Concentration & 0.09 & 0.20 & $0.11 * * *$ & 0.08 & 0.13 & $0.05 * * *$ & 0.09 & 0.12 & $0.04 * * *$ \\
\hline Existence Time & 6.19 & 13.44 & $7.26 * * *$ & 5.91 & 8.31 & $2.40 * * *$ & 6.17 & 8.08 & $1.91 * * *$ \\
\hline Darknet Sites & 17.17 & 16.67 & $-0.50 * * *$ & 17.13 & 17.17 & $0.04 * * *$ & 16.87 & 18.04 & $1.18 * * *$ \\
\hline Tumbling & 0.40 & 1.18 & $0.78 * * *$ & 0.37 & 0.64 & $0.27 * * *$ & 0.31 & 0.89 & $0.58 * * *$ \\
\hline Darknet Shock Volume & 15.84 & 27.25 & $11.40 * * *$ & 14.51 & 21.39 & $6.88 * * *$ & 10.57 & 36.14 & $25.56^{* * *}$ \\
\hline Bitcoin Hype & 28.74 & 21.16 & $-7.58 * * *$ & 29.67 & 24.95 & $-4.72 * * *$ & 30.99 & 19.38 & $-11.60 * * *$ \\
\hline Pre-Silk-Road User & 0.06 & 0.22 & $0.16 * * *$ & 0.06 & 0.12 & $0.07 * * *$ & 0.03 & 0.22 & $0.19 * * *$ \\
\hline Bitcoin Market Cap & 9.85 & 9.45 & $-0.40 * * *$ & 9.88 & 9.68 & $-0.21 * * *$ & 9.96 & 9.36 & $-0.60 * * *$ \\
\hline Shadow Coins & 7.18 & 5.34 & $-1.84 * * *$ & 7.30 & 6.51 & $-0.79 * * *$ & 7.67 & 5.11 & $-2.56 * * *$ \\
\hline Alt Coins & 8.75 & 7.49 & $-1.26 * * *$ & 8.86 & 8.24 & $-0.62 * * *$ & 9.04 & 7.47 & $-1.57 * * *$ \\
\hline
\end{tabular}


Table 6

\section{DCE model estimates}

This table reports the coefficient estimates and marginal effects of two detection-controlled estimation (DCE) models. Both models use the two-equation structure given by equations (1-4) of the paper. Model 1 is the baseline model used for the main results in the paper. Model 2 includes additional control variables. I() is the probability that a given user is predominantly using bitcoin for illegal activity. $\mathrm{D}()$ is the conditional probability of detection. Variables are defined in Table 1. Numbers not in brackets are the coefficient estimates. Numbers in brackets are the marginal effects (partial derivatives of the corresponding probability with respect to each of the variables, reported as a fraction of the estimated corresponding probability). Pseudo $R^{2}$ is McFadden's likelihood ratio index (one minus the ratio of the log-likelihood with all predictors and the log-likelihood with intercepts only). Significance at the $1 \%, 5 \%$, and $10 \%$ levels is indicated by $* * *, * *$, and $*$, respectively, using bootstrapped standard errors.

\begin{tabular}{|c|c|c|c|c|}
\hline \multirow[b]{2}{*}{ Variable } & \multicolumn{2}{|c|}{ Model 1} & \multicolumn{2}{|c|}{ Model 2} \\
\hline & $\mathrm{I}()$ & $\mathrm{D}()$ & $\mathrm{I}()$ & $\mathrm{D}()$ \\
\hline \multirow[t]{2}{*}{ Intercept } & $-1.147 * * *$ & $0.265 * * *$ & $-1.054 * * *$ & 0.066 \\
\hline & $(-0.755)$ & $(0.126)$ & $(-0.677)$ & $(0.033)$ \\
\hline \multirow[t]{2}{*}{ Darknet Sites } & $1.005 * * *$ & & $1.076 * * *$ & \\
\hline & $(0.661)$ & & $(0.691)$ & \\
\hline \multirow[t]{2}{*}{ Tumbling } & $0.085 * * *$ & & $0.103 * * *$ & \\
\hline & $(0.056)$ & & $(0.066)$ & \\
\hline \multirow[t]{2}{*}{ Bitcoin Market Cap } & $-1.608 * * *$ & & $-1.690^{* * *}$ & \\
\hline & $(-1.059)$ & & $(-1.085)$ & \\
\hline \multirow[t]{2}{*}{ Shadow Coins } & $-0.649 * * *$ & & $-0.679 * * *$ & \\
\hline & $(-0.428)$ & & $(-0.436)$ & \\
\hline \multirow[t]{2}{*}{ Alt Coins } & 0.591 & & 0.615 & \\
\hline & $(0.389)$ & & $(0.395)$ & \\
\hline \multirow[t]{2}{*}{ Darknet Shock Volume } & $0.445^{* * *}$ & & $0.496 * * *$ & \\
\hline & $(0.293)$ & & $(0.319)$ & \\
\hline \multirow[t]{2}{*}{ Pre-Silk-Road User } & & $0.430 * * *$ & & $0.430^{* *}$ \\
\hline & & $(0.204)$ & & $(0.213)$ \\
\hline \multirow[t]{2}{*}{ Transaction Frequency } & $0.438 * * *$ & $0.477 * * *$ & 0.230 & 0.474 \\
\hline & $(0.288)$ & $(0.227)$ & $(0.148)$ & $(0.235)$ \\
\hline \multirow[t]{2}{*}{ Transaction Size } & 0.005 & $-0.171 * * *$ & $-1.574 * * *$ & $-0.443 * *$ \\
\hline & $(0.003)$ & $(-0.081)$ & $(-1.011)$ & $(-0.220)$ \\
\hline \multirow[t]{2}{*}{ Concentration } & $0.293 * * *$ & $0.542 * * *$ & $0.268 * * *$ & $0.500 * * *$ \\
\hline & $(0.193)$ & $(0.258)$ & $(0.172)$ & $(0.248)$ \\
\hline \multirow[t]{2}{*}{ Existence Time } & 0.098 & $1.744 * * *$ & -0.058 & 1.405 \\
\hline & $(0.064)$ & $(0.829)$ & $(-0.037)$ & $(0.697)$ \\
\hline \multirow[t]{2}{*}{ Holding Value } & & & $3.602 * * *$ & -0.537 \\
\hline & & & $(2.312)$ & $(-0.266)$ \\
\hline \multirow[t]{2}{*}{ Transaction Count } & & & 7.900 & -0.593 \\
\hline & & & $(5.071)$ & $(-0.294)$ \\
\hline Pseudo $R^{2}$ & $21.92 \%$ & & $22.08 \%$ & \\
\hline
\end{tabular}


Table 7

Network characteristics of legal and illegal bitcoin user networks

This table reports metrics that characterize the trade networks of estimated legal and illegal bitcoin users. In the columns labelled "SLM" user classifications into legal and illegal communities are based on a network cluster analysis algorithm (SLM) and in the columns labelled "DCE" the classifications are from a detection-controlled estimation (DCE) model. Density takes the range $[0,1]$ and indicates how highly connected the users are within a community (versus how sparse the connections are between users); it is the actual number of links between users within the given community (a "link" between two users means that they have transacted with one another) divided by the total potential number of links. Reciprocity takes the range $[0,1]$ and indicates the tendency for users to engage in two-way interactions (both sending and receiving bitcoin to and from one another); it is the number of two-way links between users within the given community divided by the total number of links within the given community (two-way and one-way). Entropy measures the amount of heterogeneity among users in their number of links. It takes its minimum value of zero when all users have the same number of links (same degree).

\begin{tabular}{lcccc}
\hline Metric & \multicolumn{2}{c}{ SLM } & \multicolumn{2}{c}{ DCE } \\
\cline { 2 - 5 } & Legal & Illegal & Legal & Illegal \\
\hline Density $\left(10^{-6}\right)$ & 0.04 & 0.13 & 0.04 & 0.17 \\
Reciprocity & 0.01 & 0.03 & 0.01 & 0.03 \\
Entropy & 1.50 & 1.75 & 1.53 & 1.73 \\
\hline
\end{tabular}


Table 8

\section{Robustness tests}

This table reports robustness tests for the sensitivity of the overall estimated amount of illegal activity in bitcoin to variations in the specification of the underlying empirical models. The rows reflect estimates from different models. SLM Baseline and DCE Baseline are the SLM and DCE models used to produce the main results, and are included for comparison. The models labelled "Alternative" are variations on the corresponding baseline model. SLM Alternative 1 is an SLM model that considers the transaction volume (in bitcoins) rather than the transaction count as a measure of trading activity when applying the network cluster analysis algorithm. SLM Alternative 2 is a variation of the baseline SLM model in which observed (known) illegal users are constrained from leaving the illegal community. DCE Alternative 1, 2, and 3 are variations of the baseline DCE model in which exclusion restrictions for the instrumental variables are relaxed one at a time (these models correspond to Models 1-3 of Table A1 in the Online Appendix) respectively. The measures of group size and activity are: the number of users (Users), the number of transactions (Transaction Count), the average dollar value of bitcoin holdings (Holding Value), the number of bitcoin addresses (Number Of Addresses), and the dollar volume of transactions (Volume). Panel A reports the values of these measures for the two user groups, while Panel B expresses the measures for each group as a percentage of the total.

\begin{tabular}{|c|c|c|c|c|c|c|}
\hline Group & Model & $\begin{array}{l}\text { Users } \\
\text { (Mil) }\end{array}$ & $\begin{array}{c}\text { Transaction } \\
\text { Count } \\
\text { (Mil) }\end{array}$ & $\begin{array}{l}\text { Holding } \\
\text { Value } \\
\text { (\$Mil) }\end{array}$ & $\begin{array}{c}\text { Number Of } \\
\text { Addresses } \\
\text { (Mil) }\end{array}$ & $\begin{array}{l}\text { Volume } \\
\text { (\$Bil) }\end{array}$ \\
\hline \multicolumn{7}{|c|}{ Panel A: Values } \\
\hline \multirow[t]{7}{*}{ Illegal } & SLM Baseline & 30.94 & 276.63 & $1,394.76$ & 87.95 & 436.78 \\
\hline & SLM Alternative 1 & 28.95 & 270.69 & $1,418.42$ & 85.10 & 400.29 \\
\hline & SLM Alternative 2 & 23.68 & 287.42 & $1,866.47$ & 89.23 & 441.94 \\
\hline & DCE Baseline & 24.68 & 282.70 & $1,523.87$ & 86.35 & 422.05 \\
\hline & DCE Alternative 1 & 27.12 & 317.69 & $2,349.53$ & 98.51 & 479.13 \\
\hline & DCE Alternative 2 & 24.59 & 276.56 & $1,474.28$ & 82.47 & 420.22 \\
\hline & DCE Alternative 3 & 25.73 & 284.45 & $1,444.59$ & 87.52 & 414.52 \\
\hline \multicolumn{7}{|c|}{ Panel B: Percentages } \\
\hline \multirow[t]{7}{*}{ Illegal } & SLM Baseline & $29.12 \%$ & $45.67 \%$ & $47.05 \%$ & $39.67 \%$ & $23.45 \%$ \\
\hline & SLM Alternative 1 & $27.25 \%$ & $44.69 \%$ & $47.84 \%$ & $38.38 \%$ & $21.49 \%$ \\
\hline & SLM Alternative 2 & $22.29 \%$ & $47.45 \%$ & $62.96 \%$ & $40.25 \%$ & $23.73 \%$ \\
\hline & DCE Baseline & $23.23 \%$ & $46.67 \%$ & $51.40 \%$ & $38.95 \%$ & $22.66 \%$ \\
\hline & DCE Alternative 1 & $25.53 \%$ & $52.45 \%$ & $79.25 \%$ & $44.43 \%$ & $25.72 \%$ \\
\hline & DCE Alternative 2 & $23.14 \%$ & $45.66 \%$ & $49.73 \%$ & $37.20 \%$ & $22.56 \%$ \\
\hline & DCE Alternative 3 & $24.22 \%$ & $46.96 \%$ & $48.73 \%$ & $39.47 \%$ & $22.26 \%$ \\
\hline
\end{tabular}


Panel A: Example of illegal drugs that can be purchased with bitcoin on the Silk Road marketplace

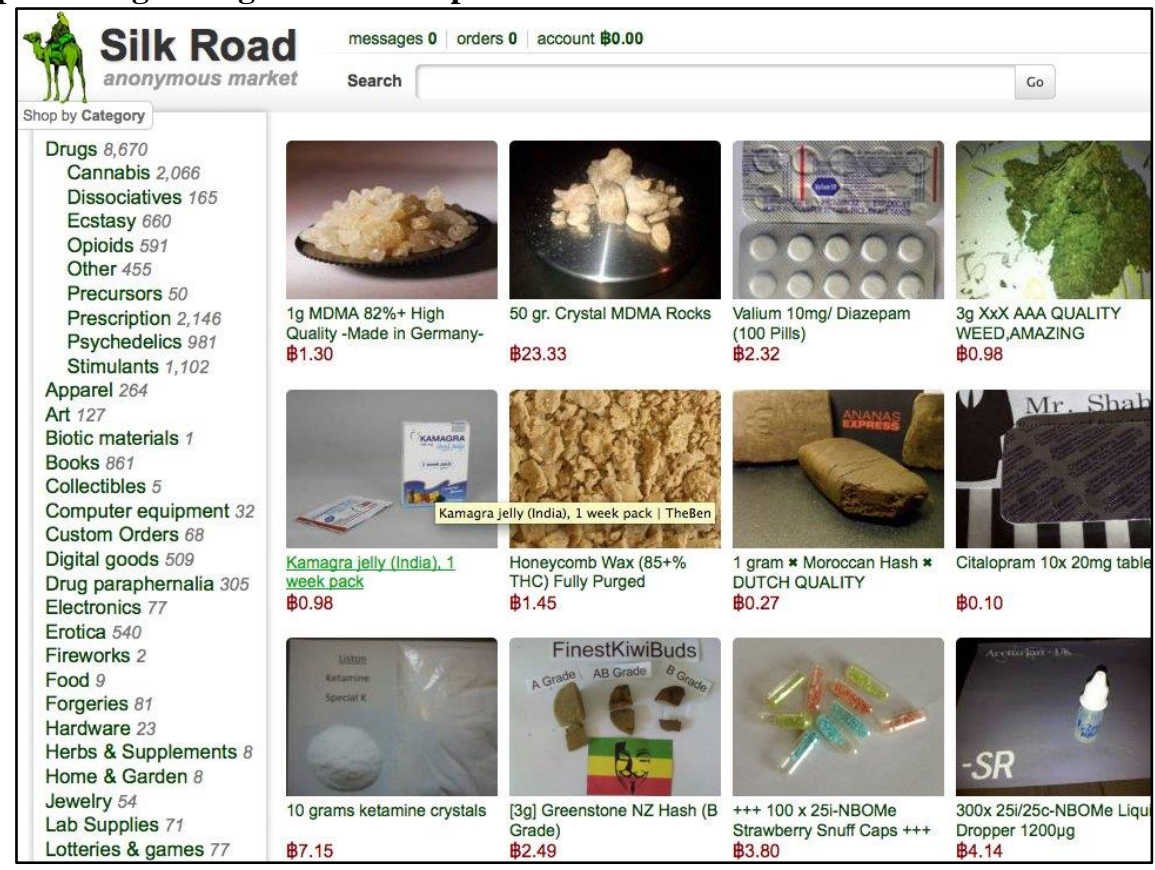

Panel B: Example of information on individual items and sellers on the Silk Road marketplace

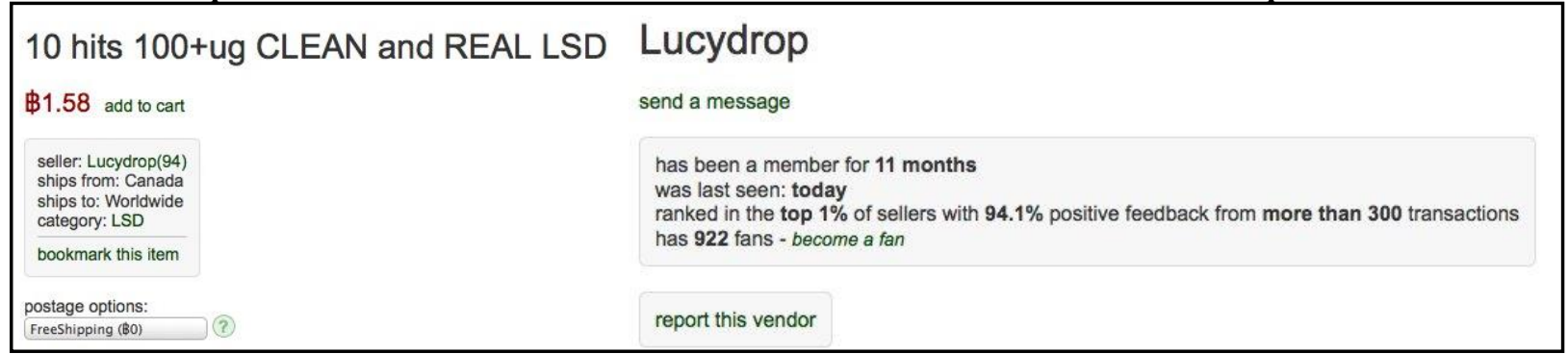

\section{Panel C: The escrow account and bitcoin payment interface for the Silk Road marketplace}

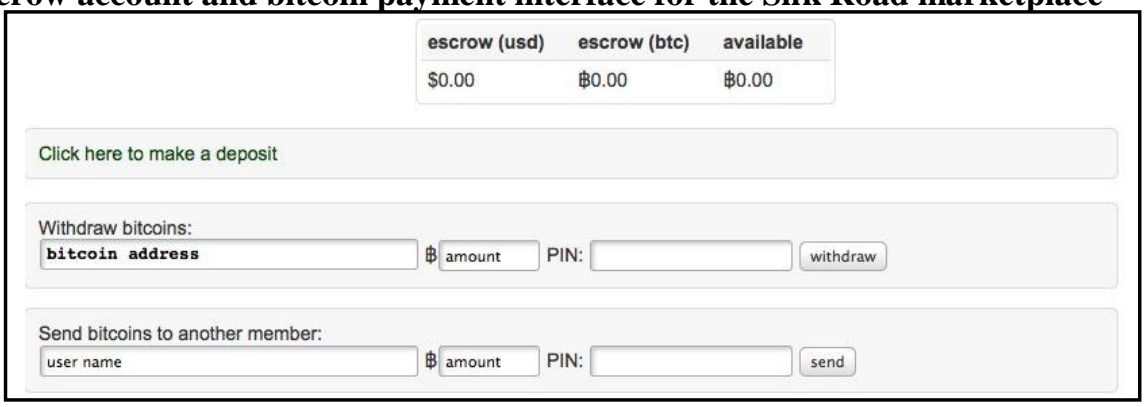

\section{Figure 1}

\section{Screenshots from one of the first illegal darknet marketplaces, Silk Road 1}

Panel A provides an example of the "Drugs" page from Silk Road. It illustrates the wide variety of illegal goods that can be purchased using bitcoin, including a vast array of illegal drugs, weapons, and forgeries. Panel B provides an example of information about individual items and sellers. Clicking on the appropriate headings, one can obtain further information about the item for sale (detailed product description, insurance/refunds, postage methods and locations, security and encryption, etc.) and about the seller (detailed feedback and ratings from buyers, history of sales, etc.). Panel C shows the interface for depositing bitcoin to Silk Road's escrow account, transferring bitcoins to a given seller, and withdrawing bitcoins from escrow. Screenshot source: www.businessinsider.com.au. 
Panel A: Percentage of users

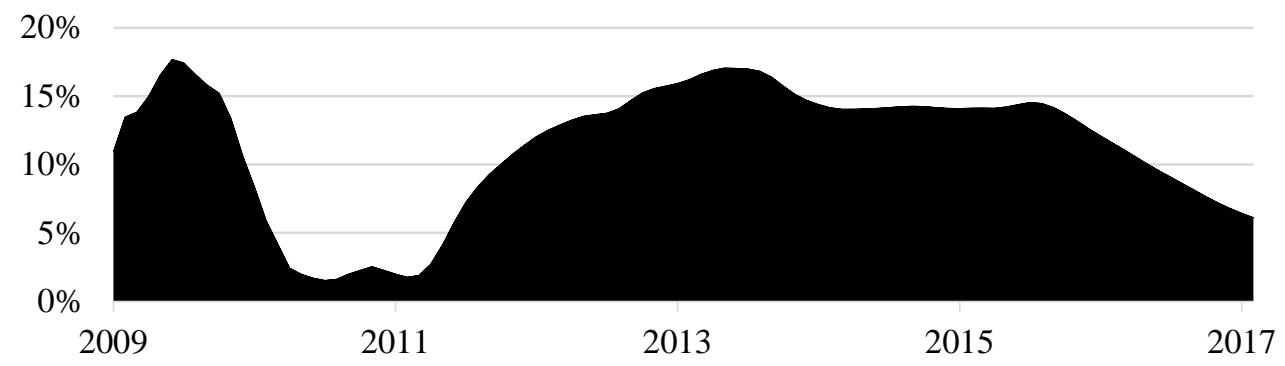

Panel B: Percentage of transactions

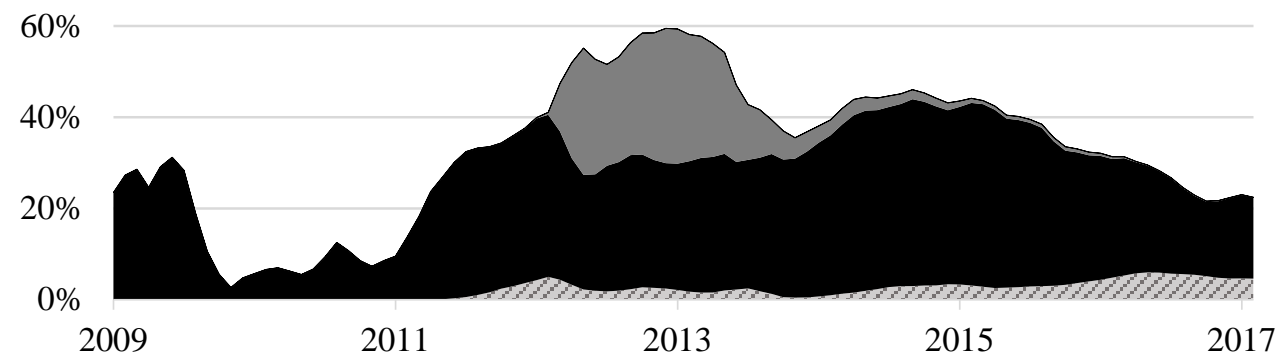

Panel C: Percentage of dollar volume

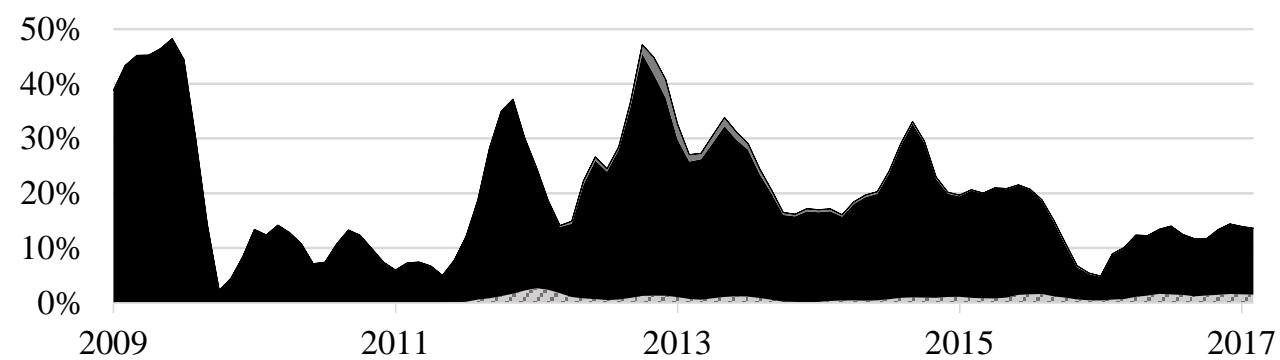

Panel D: Percentage of bitcoin holdings

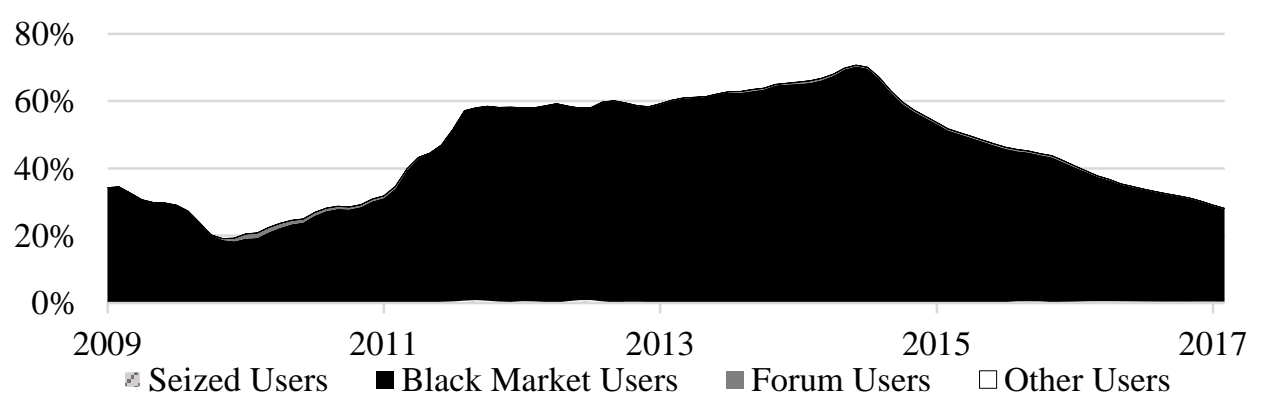

Figure 2

Size and activity of the sample of "observed" illegal bitcoin users

This figure illustrates the time-series of the three subgroups of observed illegal users as a percentage of total users (Panel A), their number of transactions as a percentage of all transaction (Panel B), the dollar value of their transactions as a percentage of the dollar value of all transactions (Panel C), and the dollar value of their bitcoin holdings as a percentage of the dollar value of all bitcoin holdings (Panel D). The observed illegal user group includes three subgroups: users that had bitcoin seized by law enforcement agencies ("Seized Users"), illegal darknet marketplace escrow accounts (hot wallets), and users that have sent or received bitcoin from those accounts ("Black Market Users"), and users whose bitcoin address(es) are mentioned in darknet forums ("Forum Users"). "Other Users" corresponds to all bitcoin users other than those in the sample of observed illegal users. The values are smoothed using a three-month moving average. 


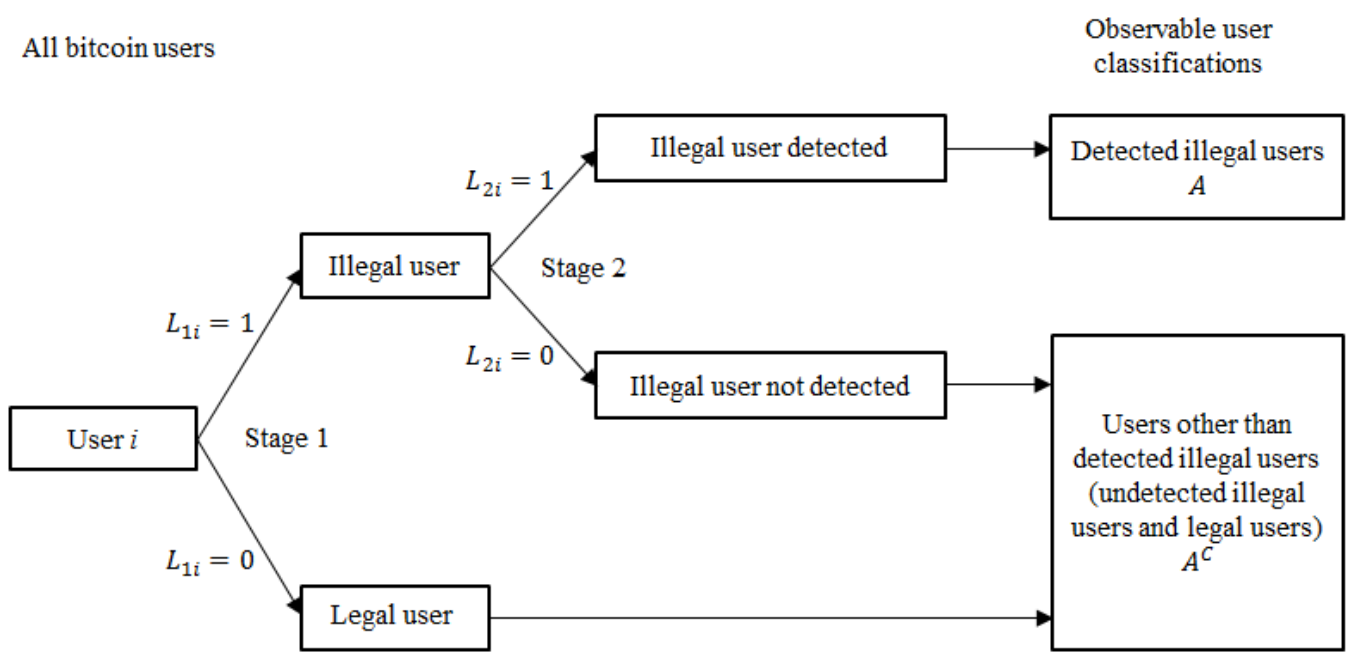

\section{Figure 3}

Two-stage detection-controlled estimation (DCE) model

The figure illustrates the structure of the two-stage DCE model. Stage 1 models how legal and illegal users of bitcoin differ in characteristics. Stage 2 models the determinants of the probability that an illegal user was "detected" (had bitcoin seized by a law enforcement agency, was identified in darknet forums, or was observed in the blockchain data as having transacted with a known illegal darknet marketplace). Both stages are estimated simultaneously using maximum likelihood to select parameter values that maximize the likelihood of the observable user classifications, $A$ and $A^{C}$. 
Panel A: Estimated number of illegal and legal bitcoin users

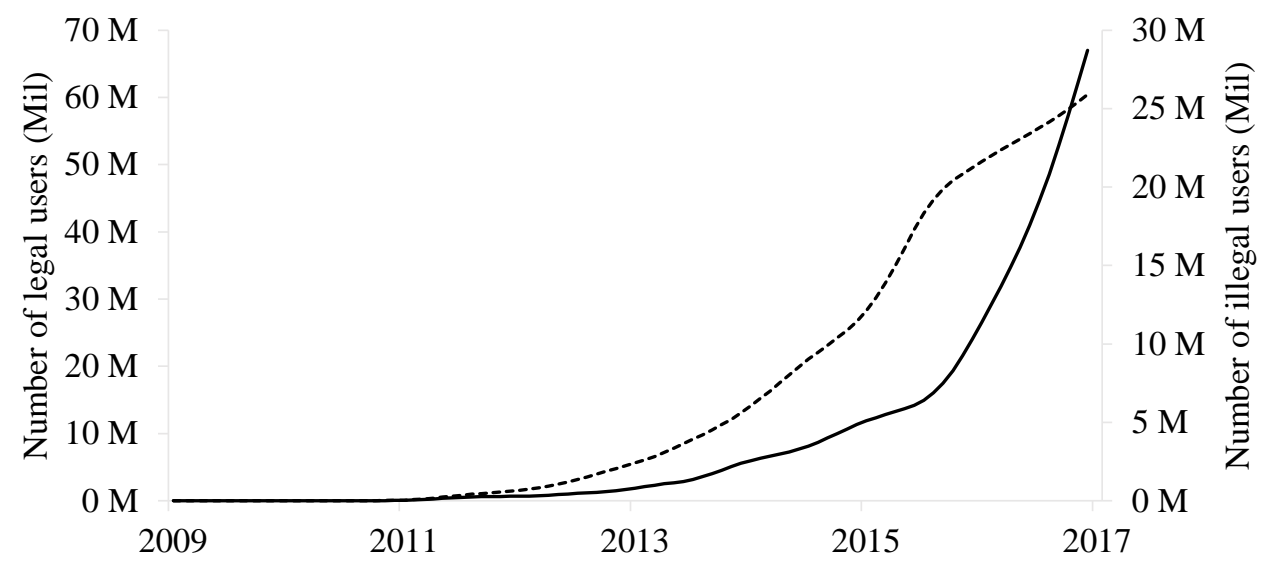

Panel B: Estimated percentage of illegal bitcoin users with 99\% confidence bounds

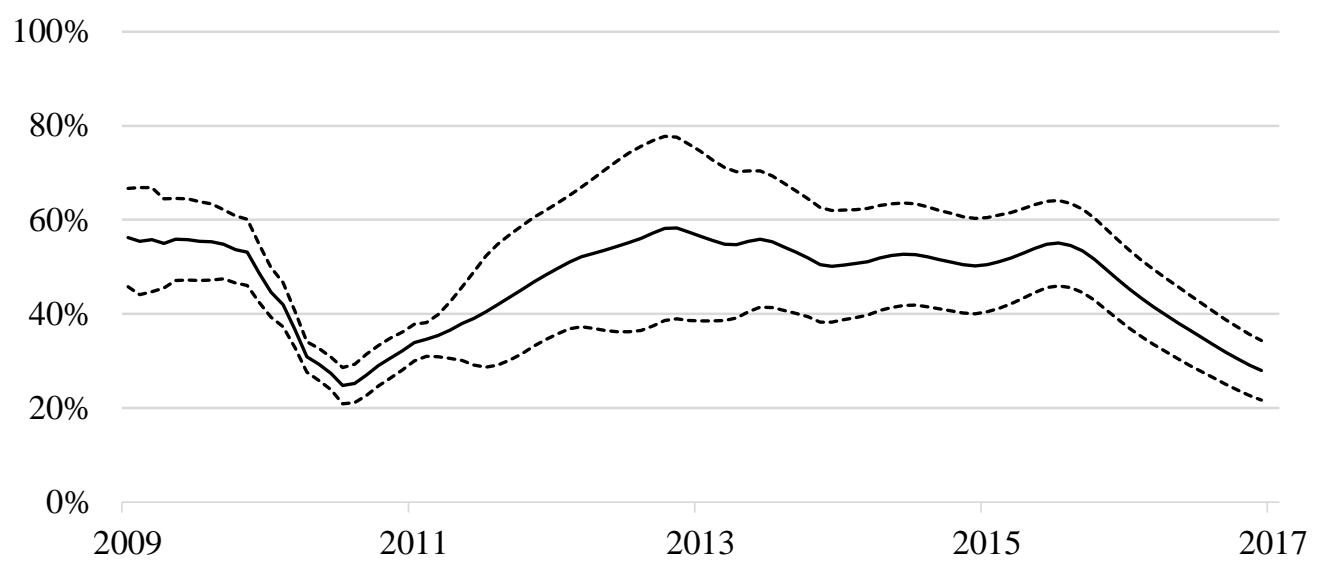

\section{Figure 4}

\section{Estimated number and percentage of bitcoin users involved in illegal activity}

This figure illustrates the time-series of the estimated number of illegal and legal bitcoin users (Panel A) and the percentage of illegal users (Panel B). In Panel A, the number of legal users is plotted with the solid line using the left-hand-side axis and the number of illegal users is plotted with the dashed line using the right-hand-side axis. In Panel B, the solid line is the point estimate of the percentage of illegal users and the dashed lines provide a $99 \%$ confidence interval using bootstrapped standard errors. The estimates come from a combination of two empirical models (the average of the estimates produced by the SLM and DCE models). Values are smoothed using a moving average. 


\section{Panel A: Estimated number of illegal and legal bitcoin user transactions per month}

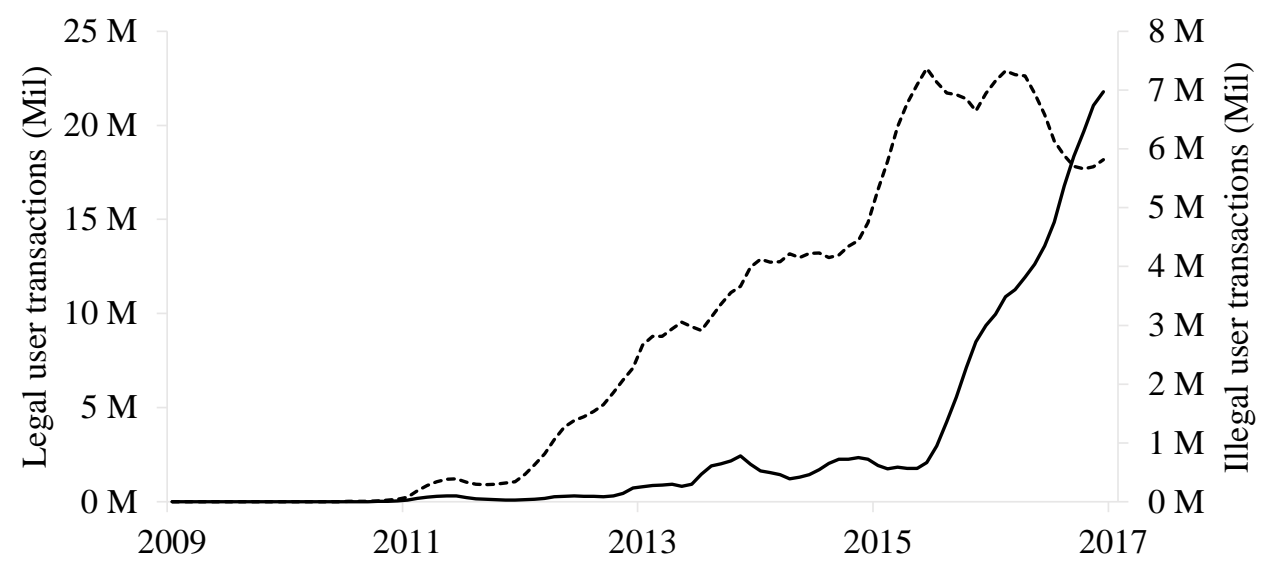

Panel B: Estimated percentage illegal user transactions with 99\% confidence bounds

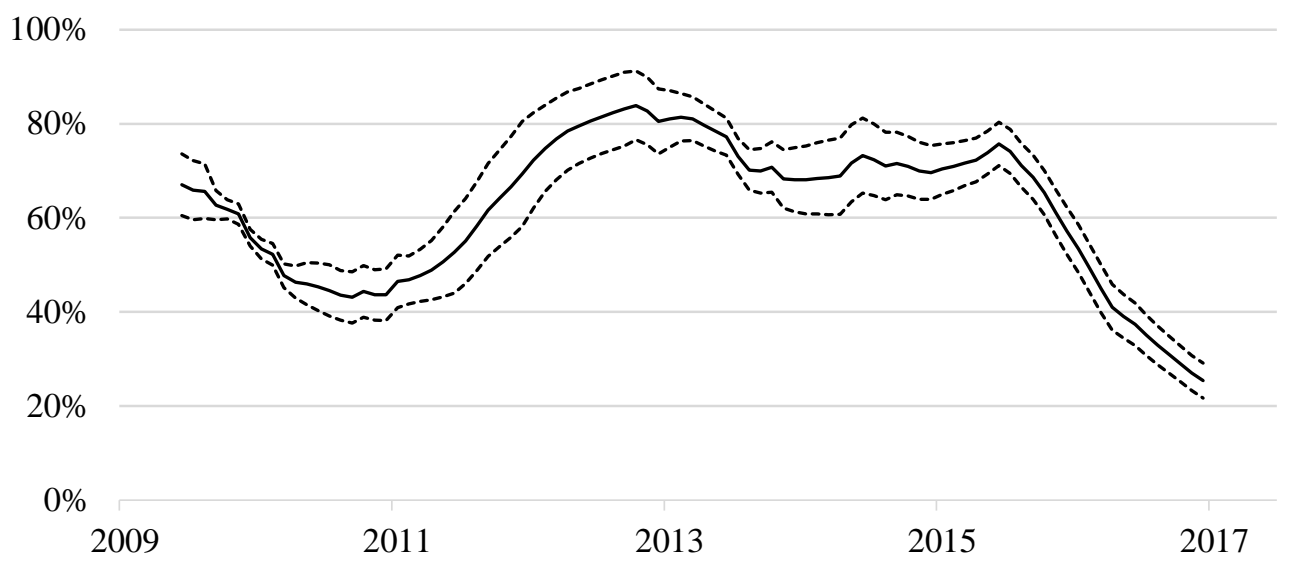

\section{Figure 5}

Estimated number and percentage of illegal bitcoin users transactions

This figure illustrates the time-series of the estimated number of illegal and legal bitcoin user transactions per month (Panel A) and the percentage of illegal user transactions (Panel B). In Panel A, the number of legal user transactions is plotted with the solid line using the left-hand-side axis and the number of illegal user transactions is plotted with the dashed line using the right-hand-side axis. In Panel B, the solid line is the point estimate of the percentage of illegal user transactions and the dashed lines provide a $99 \%$ confidence interval using bootstrapped standard errors. The estimates come from a combination of two empirical models (the average of the estimates produced by the SLM and DCE models). Values are smoothed using a moving average. 


\section{Panel A: Estimated dollar volume of illegal and legal bitcoin user transactions per month}

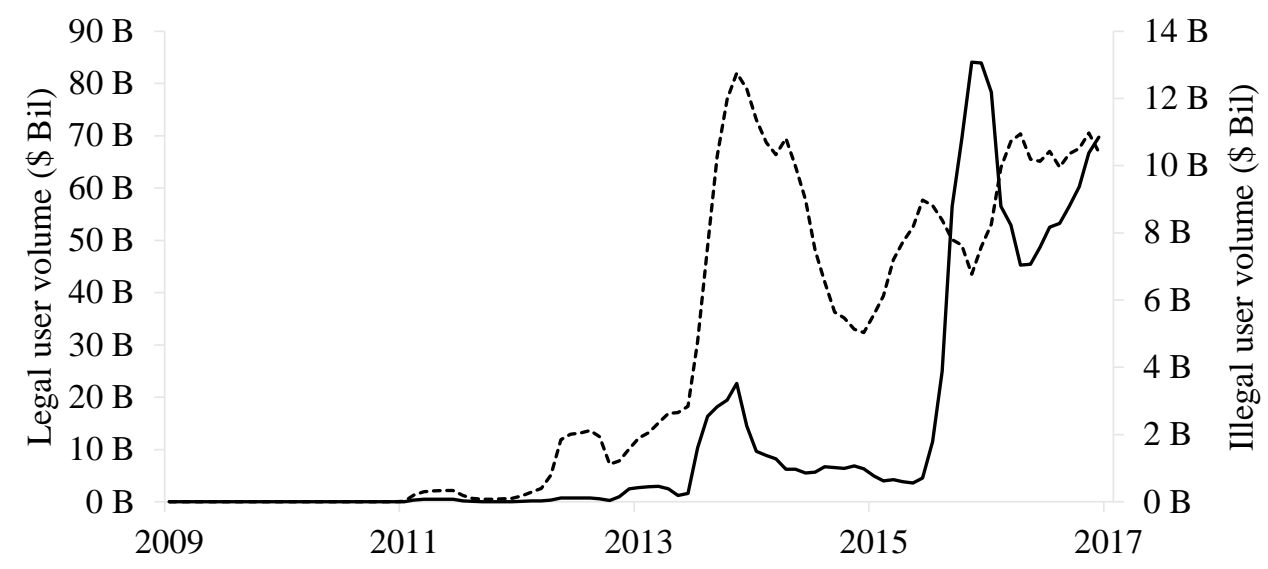

Panel B: Estimated percentage illegal user dollar volume with 99\% confidence bounds

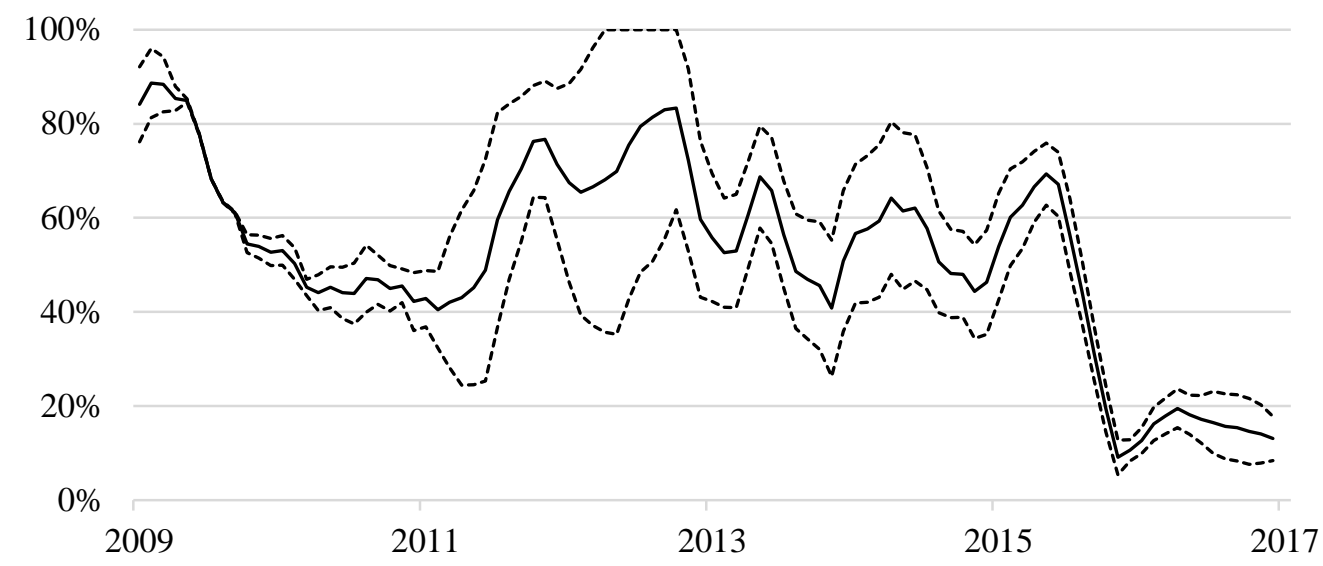

\section{Figure 6}

Estimated dollar volume and percentage dollar volume of illegal bitcoin user transactions

This figure illustrates the time-series of the estimated dollar volume of illegal and legal bitcoin user transactions per month (Panel A) and illegal user dollar volume as a percentage of total dollar volume of bitcoin transactions (Panel B). In Panel A, the dollar volume of legal user transactions is plotted with the solid line using the left-hand-side axis and the dollar volume of illegal user transactions is plotted with the dashed line using the right-hand-side axis. In Panel B, the solid line is the point estimate of the illegal dollar volume as a percentage of total dollar volume and the dashed lines provide a 99\% confidence interval using bootstrapped standard errors. The estimates come from a combination of two empirical models (the average of the estimates produced by the SLM and DCE models). Values are smoothed using a moving average. 
Panel A: Estimated dollar value of illegal and legal user bitcoin holdings

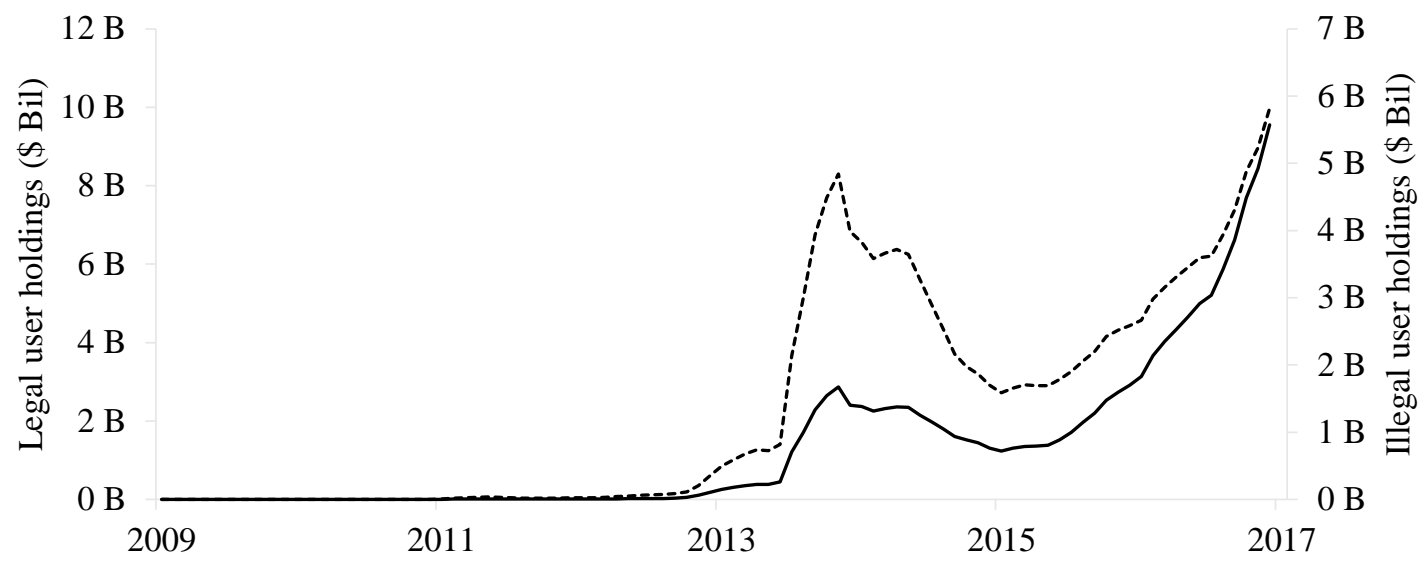

Panel B: Estimated percentage of illegal users bitcoin holdings with 99\% confidence bounds

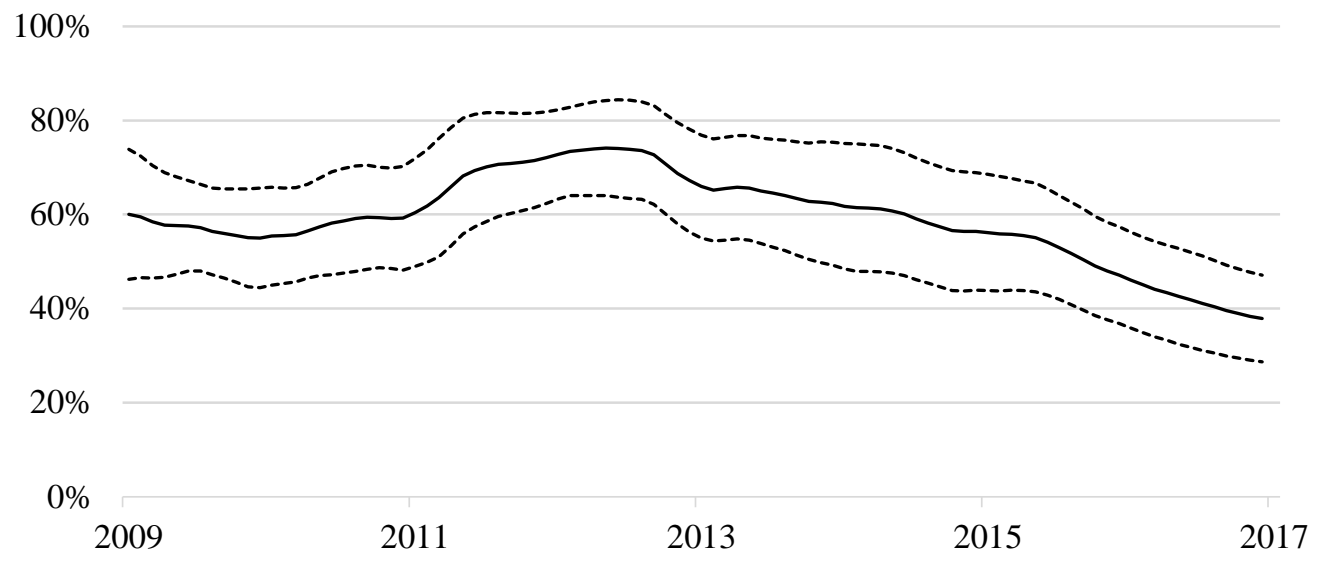

Figure 7

Estimated dollar value and percentage of illegal user bitcoin holdings

This figure illustrates the time-series of the estimated dollar value of illegal and legal user bitcoin holdings (Panel A) and illegal user holdings as a percentage of total bitcoin holdings (Panel B). In Panel A, the dollar value of legal user bitcoin holdings is plotted with the solid line using the left-hand-side axis and the dollar value of illegal user holdings is plotted with the dashed line using the right-hand-side axis. In Panel B, the solid line is the point estimate of the illegal user holdings as a percentage of total bitcoin holdings and the dashed lines provide a $99 \%$ confidence interval using bootstrapped standard errors. The estimates come from a combination of two empirical models (the average of the estimates produced by the SLM and DCE models). Values are smoothed using a moving average. 